\title{
Global analysis of hadronic two-body $B$ decays in the perturbative QCD approach
}

\author{
Jun Hua $\odot,{ }^{1, \dagger}$ Hsiang-nan Li®${ }^{2, \ddagger}$ Cai-Dian Lü, ${ }^{3,4, \S}$ Wei Wang $\odot,{ }^{1, *}$ and Zhi-Peng Xing ${ }^{1, \|}$ \\ ${ }^{1}$ INPAC, Key Laboratory for Particle Astrophysics and Cosmology (MOE), \\ Shanghai Key Laboratory for Particle Physics and Cosmology, \\ School of Physics and Astronomy, Shanghai Jiao-Tong University, \\ Shanghai 200240, China \\ ${ }^{2}$ Institute of Physics, Academia Sinica, Taipei, Taiwan 11529, Republic of China \\ ${ }^{3}$ Institute of High Energy Physics, Chinese Academy of Sciences, Beijing 100049, China \\ ${ }^{4}$ School of Physics, University of Chinese Academy of Sciences, Beijing 100049, China
}

(Received 7 January 2021; accepted 8 July 2021; published 28 July 2021)

\begin{abstract}
Based on the flavor structure of four-quark effective operators, we develop an automatic computation program to calculate hadronic two-body $B$ meson decay amplitudes, and apply it to their global analysis in the perturbative QCD (PQCD) approach. Fitting the PQCD factorization formulas for $B \rightarrow P P, V P$ decays at leading order in the strong coupling $\alpha_{s}$ to measured branching ratios and direct $C P$ asymmetries, we determine the Gegenbauer moments in light meson light cone distribution amplitudes (LCDAs). It is found that most of the fitted Gegenbauer moments of the twist-2 and twist-3 LCDAs for the pseudoscalar meson $P(P=\pi, K)$ and vector meson $V\left(V=\rho, K^{*}\right)$ agree with those derived in QCD sum rules. The shape parameter for the $B_{s}$ meson distribution amplitude and the weak phase $\phi_{3}(\gamma)=(75.2 \pm 2.9)^{\circ}$ consistent with the value in Particle Data Group are also obtained. It is straightforward to extend our analysis to higher orders and higher powers, when the corresponding subleading contributions are available in the PQCD approach, and to the global determination of LCDAs for other hadrons.
\end{abstract}

DOI: 10.1103/PhysRevD.104.016025

\section{INTRODUCTION}

The study of heavy quark physics is firmly in the precision era nowadays. On the experimental side, the $B$ factories, i.e., the $B A B A R$, Belle, and LHCb, have collected abundant data of exclusive $B$ meson decays, which can be employed not only to explore involved rich QCD dynamics but also to probe the origin of $C P$ violation and potential new physics signals [1]. Vastly more data will be still accumulated by the upgraded LHCb and Belle-II Collaborations [2-4]. On the theoretical side, tremendous progress on the development of QCD treatments of exclusive $B$ meson decays with controllable uncertainties has been achieved. Strict confrontation between data and theoretical expectations has led to some mild tensions

\footnotetext{
* Corresponding author. wei.wang@sjtu.edu.cn

thuajun_phy@sjtu.edu.cn

thnli@phys.sinica.edu.tw

\$lucd@ihep.ac.cn

"zpxing@sjtu.edu.cn
}

Published by the American Physical Society under the terms of the Creative Commons Attribution 4.0 International license. Further distribution of this work must maintain attribution to the author(s) and the published article's title, journal citation, and DOI. Funded by SCOAP ${ }^{3}$. between experimental observations and the Standard Model [1], which may be vaguely attributed to new physics beyond the Standard Model. This undoubtedly motivates the attempt to gain deeper understanding of QCD dynamics in exclusive $B$ meson decays and better control of hadronic uncertainties.

The $b$ quark mass $m_{b}$, much larger than the QCD hadronic scale $\Lambda_{\mathrm{QCD}}$, renders QCD analyses of exclusive $B$ meson decays possible. Nonperturbative dynamics in heavy meson decays is reflected by infrared divergences in radiative corrections. When a factorization theorem holds, infrared divergences are absorbed into hadron light cone distribution amplitudes (LCDAs), so that the remnant, being infrared finite, is calculable at the parton level in perturbation theory. A physical quantity, such as a heavyto-light transition form factor, is then factorized into a convolution of a $b$ quark decay hard kernel with hadron LCDAs in parton momentum fractions. The corresponding factorization theorem should be proved to all orders in the strong coupling $\alpha_{s}$ and to certain power in $\Lambda_{\mathrm{QCD}} / m_{b}$. LCDAs, despite of being nonperturbative, are universal, i.e., process independent. With this universality, LCDAs, determined by nonperturbative methods like QCD sum rules [5-7] and lattice QCD [8-10], or extracted from experimental data, can be employed to make predictions for other modes involving the same hadrons. 
The theoretical approaches based on factorization theorems in the heavy quark limit include light cone QCD sum rules (LCSR) [11-13], the QCD-improved factorization (QCDF) [14], the perturbative QCD (PQCD) factorization [15-20], and the soft-collinear effective theory (SCET) $[21,22]$. The collinear factorization applies to relevant correlators in LCSR, where some hadronic states are expanded into parton Fock states characterized by different twists. The QCDF approach is an extension of the naive factorization assumption for hadronic two-body $B$ meson decays in the collinear factorization theorem. The SCET for kinematic regions with energetic final state hadrons is equivalent to the collinear factorization theorem, but formulated in terms of effective operators. The $k_{T}$ factorization theorem is the basis of the PQCD approach, which is more appropriate in the endpoint region of parton momentum fractions. Many efforts have been devoted to systematic investigation of hadronic two-body $B$ meson decays at various orders in $\alpha_{s}$ and powers in $\Lambda_{\mathrm{QCD}} / m_{b}$ [23-25]. In all the above formalisms nonperturbative hadron LCDAs provide one of major sources of theoretical uncertainties.

A hadron LCDA can be expanded into a series of Gegenbauer polynomials with the coefficients, namely, the Gegenbauer moments being determined by other methods as aforementioned. Though some attempts have been made to calculate Gegenbauer moments using lattice QCD [8-10], not all LCDAs are constrained in this way so far. Here we will perform a global fit of the Gegenbauer moments in light meson LCDAs to measured branching ratios and direct $C P$ asymmetries in hadronic two-body $B$ meson decays in the PQCD approach. It is known that enormous efforts have been devoted for several decades to global determinations of parton distribution functions from experimental data, which serve as the nonperturbative inputs to the factorization of inclusive QCD processes. What we are proposing, i.e., the global fit of hadron LCDAs, is a parallel version for exclusive processes. We believe that it is time to initiate such a pronounced program, and the global analysis of hadronic two-body $B$ meson decays represents the first attempt along this line.

The involved decay amplitudes at leading order (LO) of the strong coupling $\alpha_{s}$ will be constructed automatically with a computation program by making use of flavor SU(3) properties. We establish a Gegenbauer-momentindependent database, by means of which each decay amplitude is expressed as a combination of the relevant Gegenbauer moments and Cabibbo-Kobayashi-Maskawa (CKM) matrix elements. The Gegenbauer moments in the leading-twist (twist-2) and next-to-leading-twist (twist-3) LCDAs for the pseudoscalar meson $P(P=\pi, K)$ and vector meson $V\left(V=\rho, K^{*}\right)$ are then fixed in the global fit, most of which are found to agree with those from QCD sum rules [5-7]. It should be noticed that the precision of extracted LCDAs depends on the accuracy of the involved hard kernels. As a by-product, the shape parameter for the
$B_{s}$ meson distribution amplitude (DA) and the weak phase $\phi_{3}(\gamma)=(75.2 \pm 2.9)^{\circ}$ consistent with the value in Particle Data Group [26] are also obtained. Though we have focused on the $B \rightarrow P P, V P$ decays at $\mathrm{LO}$ in PQCD, our work provides the first setup for a global analysis of exclusive $B$ meson decays, and can be generalized straightforwardly to include other modes, and higher-order and/or higher-power corrections, when they are available.

The rest of this paper is organized as follows. We give a brief overview of the theoretical framework for hadronic two-body $B$ meson decays in Sec. II. The automatic derivation of the decay amplitudes in the PQCD approach is formulated in Sec. III, where the Gegenbauer-momentindependent database for the considered modes is established. We perform a global fit of meson LCDAs and the CKM angle $\phi_{3}(\gamma)$ to a limited number of physical observables in the $B \rightarrow P P, V P$ decays, and present the numerical results in Sec. IV. We also compare our predictions for some other modes excluded in the fit with experimental data. A few remarks and future improvements on our analysis are outlined at the end of this section. Section V contains a summary of the present work. The explicit factorization formulas and their ingredients are collected in the Appendix.

\section{THEORETICAL FORMALISM}

In exclusive processes, such as heavy-to-light transition form factors, the range of a parton momentum fraction $x$, contrary to that in an inclusive case, is not experimentally controllable, and runs from 0 to 1 . Hence, the end point region with $x \rightarrow 0$ is unavoidable. If no end point singularity is developed, implying that the end point region is likely power suppressed, the collinear factorization will work. If such a singularity occurs, the collinear factorization will break down, and the $k_{T}$ factorization should be adopted. In fact, the observation $Q F_{2}\left(Q^{2}\right) / F_{1}\left(Q^{2}\right) \sim$ const. [27,28], $F_{1}$ and $F_{2}$ being the proton Dirac and Pauli form factors, respectively, and $Q$ being a momentum transferred, indicates that the $k_{T}$ factorization is an appropriate tool for studying exclusive processes [29]. It has been shown that infrared divergences appearing in loop corrections to exclusive processes can be absorbed into hadron LCDAs in the $k_{T}$ factorization without breaking the gauge invariance [30]. Since the $k_{T}$ factorization theorem was proposed [31,32], there had been broad applications to various processes [33].

The application of the collinear factorization theorem to exclusive $B$ meson decays, for instance, the $B \rightarrow \pi$ transition form factors, suffers the end point singularities mentioned above [34-36]: the twist 2 and twist-3 contributions are logarithmically and linearly divergent, respectively. The inclusion of parton transverse momenta $k_{T}$, regulating the end point singularities, induces soft logarithms in higher-order corrections. Their overlap with the existent collinear logarithms generates the double 
logarithms $\alpha_{s} \ln ^{2} k_{T}$, which must be organized in order not to spoil perturbative expansion. The basic idea for the $k_{T}$ resummation of the double logarithms into a Sudakov factor has been elaborated in [15-17,31,37], where the explicit expressions of the Sudakov exponents can be found. The resultant Sudakov suppression on the low $k_{T}$ contribution in the end point region renders the magnitude of $k_{T}^{2}$ roughly $O\left(m_{b} \Lambda_{\mathrm{QCD}}\right)$. The coupling constant $\alpha_{s}\left(\sqrt{m_{b} \Lambda_{\mathrm{QCD}}}\right) / \pi \sim 0.13$ is then small enough to justify the perturbative evaluation of heavy-to-light transition form factors at large recoil $[18,38,39]$.

On the other hand, the double logarithms $\alpha_{s} \ln ^{2} x$ from radiative corrections were observed in the semileptonic decay $B \rightarrow \pi l \nu$ [40] and in the radiative decay $B \rightarrow \gamma l \nu$ [41]. It has been argued that when the end point region is important, these double logarithms should be organized into a quark jet function systematically in order to improve perturbative expansion. The procedure is referred to as the threshold resummation [42]. The resultant jet function has been shown to vanish quickly as $x \rightarrow 0$. It turns out that in a self-consistent perturbative evaluation of the heavy-to-light transition form factors, where the original factorization formulas are further convoluted with the jet function, the end point singularities do not exist [42]. The threshold resummation for the jet function has been pushed to the next-to-leading-logarithm accuracy recently [43]. Note that either the threshold or $k_{T}$ resummation smears the end point singularities. To suppress the soft contribution sufficiently, both resummations are required, such that reliable results for the heavy-to-light transition form factors can be attained.

We emphasize that the power counting for a parton transverse momentum $k_{T}$ is nontrivial, compared to the power counting for the fixed scales like $m_{b}$ and $\Lambda_{\mathrm{QCD}}$. The $k_{T}$ factorization is suitable for a multiscale process, like a heavy-to-light transition form factor, to which the region of a small momentum fraction $x$ dominates. The small $x$ introduces an additional intermediate scale $x m_{b}^{2} \sim m_{b} \Lambda_{\mathrm{QCD}}$, respecting the hierarchy $m_{b}^{2} \gg x m_{b}^{2} \gg \Lambda_{\mathrm{QCD}}^{2}$. A parton $k_{T}$, being an integration variable in a $k_{T}$ factorization formula, can take values of orders of the above scales. The $k_{T}$ factorization should apply, as a hard kernel depends on the large scale $m_{b}^{2}$ and the intermediate scale $m_{b} \Lambda_{\mathrm{QCD}}$, but not on the small scale $\Lambda_{\mathrm{QCD}}^{2}$, and the factorization of hadron wave functions hold for a parton $k_{T}$ at both the intermediate and small scales. Once these criteria are satisfied, the $k_{T}$ dependence in a hard kernel is not negligible [30], and a convolution between the hard kernel and the wave functions in $k_{T}$ is demanded. If a hard kernel involves only the large scale, the $k_{T}$ dependence of the hard kernel can be neglected. It is then integrated out in the wave functions, and one is led to the collinear factorization.

Since a wave function contains the contributions characterized by both the intermediate and small scales, it is legitimate to further factorize the former out of the wave function, as the intermediate scale is regarded as being perturbative. This gives the aforementioned $k_{T}$ resummation, which is justified perturbatively for the scale $k_{T}^{2} \sim m_{b} \Lambda_{\mathrm{QCD}}$. After this organization, the remaining piece, i.e., the initial condition for the Sudakov resummation, involves only the small scale $\Lambda_{\mathrm{QCD}}^{2}$, and corresponds to a hadron DA. Note that a more sophisticated formalism, called the joint resummation, which organizes the mixed logarithms formed by the above two different scales, has been developed in [44]. Similarly, it is also legitimate to further factorize the contribution characterized by an intermediate scale out of a hard kernel in the $k_{T}$ factorization. This refactorization yields the jet function, through which the logarithms of $x m_{b}^{2}$ are resummed to all orders.

The effective Hamiltonian for hadronic two-body $B$ meson decays is given by [45]

$$
\begin{aligned}
\mathcal{H}_{\text {eff }}= & \frac{G_{F}}{\sqrt{2}}\left\{\sum_{q=u, c} V_{q b} V_{q D}^{*}\left[C_{1} O_{1}^{q}+C_{2} O_{2}^{q}\right]\right. \\
& \left.-V_{t b} V_{t D}^{*} \sum_{i=3}^{10} C_{i} O_{i}\right\}+ \text { H.c. },
\end{aligned}
$$

with the Fermi constant $G_{F}$, the CKM matrix elements $V_{q b(D)}, D=d, s$, the local four-quark operators $O_{i}$, and the Wilson coefficients $C_{i}$. All the factorizable, nonfactorizable and power-suppressed annihilation contributions resulting from the above four-quark operators are calculable in the PQCD approach without the end point singularities. The arbitrary cutoffs introduced in QCDF [46,47] are not necessary, and PQCD factorization formulas involve only universal and controllable inputs. The $B \rightarrow M_{2} M_{3}$ decay amplitude is generically factorized into the convolution of the Wilson coefficient $C$, a six-quark hard kernel $H$, the jet function $J_{t}$, and the Sudakov factor $S$ with meson LCDAs $\phi$ [48-51],

$$
A=\phi_{B} \otimes C \otimes H \otimes J_{t} \otimes S \otimes \phi_{M_{2}} \otimes \phi_{M_{3}},
$$

all of which are well defined and gauge invariant. The partition of nonperturbative and perturbative contributions depends on factorization schemes. However, a decay amplitude, as a convolution of the above factors, is independent of factorization schemes in principle.

\section{DATABASE FOR GLOBAL FIT}

\section{A. Light cone distribution amplitudes}

The momenta $p_{B}, p_{2}$, and $p_{3}$ of the $\bar{B}$ meson, emitted meson $M_{2}$, and recoiling meson $M_{3}$, respectively, and their associated parton momenta are chosen, in the light cone coordinates, as 


$$
\begin{aligned}
p_{B} & =\frac{m_{B}}{\sqrt{2}}\left(1,1,0_{T}\right), & k_{1} & =\left(x_{1} \frac{m_{B}}{\sqrt{2}}, 0, k_{1 T}\right), \\
p_{2} & =\frac{m_{B}}{\sqrt{2}}\left(1,0,0_{T}\right), & k_{2} & =\left(x_{2} \frac{m_{B}}{\sqrt{2}}, 0, k_{2 T}\right), \\
p_{3} & =\frac{m_{B}}{\sqrt{2}}\left(0,1,0_{T}\right), & k_{3} & =\left(0, x_{3} \frac{m_{B}}{\sqrt{2}}, k_{3 T}\right),
\end{aligned}
$$

which are labeled in Fig. 1 with $m_{B}$ being the $B$ meson mass and $x_{i}$ being the momentum fractions. The light meson LCDAs are defined through the matrix elements

$$
\begin{aligned}
\left\langle P(p)\left|q_{1 \alpha}(0) \bar{q}_{2 \beta}(z)\right| 0\right\rangle & =\frac{i}{\sqrt{2 N_{c}}} \int_{0}^{1} d x e^{i x p \cdot z}\left[\gamma_{5} \not p \phi^{A}(x)+\gamma_{5} m_{0} \phi^{P}(x)+m_{0} \gamma_{5}(\not \not h-1) \phi^{T}(x)\right]_{\alpha \beta}, \\
\left\langle V\left(p, \epsilon_{L}^{*}\right)\left|q_{1 \alpha}(0) \bar{q}_{2 \beta}(z)\right| 0\right\rangle & =\frac{-1}{\sqrt{2 N_{c}}} \int_{0}^{1} d x e^{i x p \cdot z}\left[m_{V} \phi_{L}^{*} \phi_{V}(x)+\phi_{L}^{*} \not p \phi_{V}^{t}(x)+m_{V} \phi_{V}^{s}(x)\right]_{\alpha \beta},
\end{aligned}
$$

where $N_{c}=3$ is the number of colors, $m_{0}$ is the chiral enhancement scale for the pseudoscalar meson $P$, the dimensionless vector $v=\sqrt{2} p / M_{B}$ lies in the direction of the meson momentum $p$, the dimensionless vector $n$ lies in the direction of the quark coordinate $z$ with $n \cdot v=1$, and $m_{V}$ $\left(\epsilon_{L}\right)$ is the mass (longitudinal polarization vector) of the vector meson $V$. The light meson LCDAs are expanded as

$$
\begin{aligned}
\phi_{P}(x)= & \frac{f_{P}}{2{\sqrt{2 N_{c}}}^{\prime}} 6 x(1-x)\left[1+a_{1}^{f} C_{1}^{3 / 2}(1-2 x)\right. \\
& \left.+a_{2}^{f} C_{2}^{3 / 2}(1-2 x)+a_{4}^{f} C_{4}^{3 / 2}(1-2 x)\right] \\
\phi_{P}^{P}(x)= & \frac{f_{P}}{2 \sqrt{2 N_{c}}}\left[1+a_{P 2}^{f} C_{2}^{1 / 2}(1-2 x)+a_{P 4}^{f} C_{4}^{1 / 2}(1-2 x)\right] \\
\phi_{P}^{T}(x)= & -\frac{f_{P}}{2 \sqrt{2 N_{c}}}\left[C_{1}^{1 / 2}(1-2 x)+a_{T 2}^{f} C_{3}^{1 / 2}(1-2 x)\right] \\
\phi_{V}(x)= & \frac{f_{V}}{2 \sqrt{2 N_{c}}} 6 x(1-x)\left[1+a_{1}^{f \|} C_{1}^{3 / 2}(1-2 x)\right. \\
& \left.+a_{2}^{f \|} C_{2}^{3 / 2}(1-2 x)\right] \\
\phi_{V}^{t}(x)= & \frac{3 f_{V}^{T}}{2{\sqrt{2 N_{c}}}^{2}}(1-2 x)^{2} \\
\phi_{V}^{s}(x)= & \frac{3 f_{V}^{T}}{2{\sqrt{2 N_{c}}}^{2}}(1-2 x)
\end{aligned}
$$

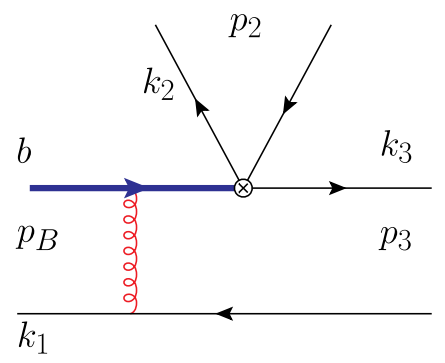

FIG. 1. A leading order Feynman diagram for the $\bar{B}\left(p_{B}\right) \rightarrow$ $M_{2}\left(p_{2}\right) M_{3}\left(p_{3}\right)$ decay. in terms of the orthogonal Gegenbauer polynomials

$C_{1}^{1 / 2}(t)=t, \quad C_{2}^{1 / 2}(t)=\frac{1}{2}\left(3 t^{2}-1\right), \quad C_{3}^{1 / 2}(t)=\frac{1}{2} t\left(5 t^{2}-3\right)$,

$C_{1}^{3 / 2}(t)=3 t, \quad C_{2}^{3 / 2}(t)=\frac{3}{2}\left(5 t^{2}-1\right)$,

$C_{4}^{3 / 2}(t)=\frac{15}{8}\left(1-14 t^{2}+21 t^{4}\right)$,

where the decay constants $f_{P}, f_{V}$ and $f_{V}^{T}$ can be extracted from leptonic decay widths such as $\Gamma(\pi \rightarrow \mu \nu)$ and $\Gamma(\tau \rightarrow \rho \nu)$, and the superscripts $f$ of the Gegenbauer moments label the species of mesons.

The $B$ meson DA is defined via the matrix element

$$
\begin{aligned}
\int & \frac{d^{4} z}{(2 \pi)^{4}} e^{i k \cdot z}\left\langle 0\left|b_{\alpha}(0) \bar{q}_{\beta}(z)\right| \bar{B}\left(p_{B}\right)\right\rangle \\
& =\frac{i}{\sqrt{2 N_{c}}}\left\{\left(\not \not_{B}+m_{B}\right) \gamma_{5}\left[\phi_{B}(k)-\frac{\not h_{+}-\not h_{-}}{\sqrt{2}} \bar{\phi}_{B}(k)\right]\right\}_{\alpha \beta},
\end{aligned}
$$

with the light spectator momentum $k$, and the dimensionless vectors $n_{+}=\left(1,0, \mathbf{0}_{T}\right)$ and $n_{-}=\left(0,1, \mathbf{0}_{T}\right)$. In this work we adopt the model for the $B_{(s)}$ meson DA,

$\phi_{B_{(s)}}(x, b)=N_{B_{(s)}} x^{2}(1-x)^{2} \exp \left[-\frac{m_{B_{(s)}}^{2} x^{2}}{2 \omega_{B_{(s)}}^{2}}-\frac{1}{2} \omega_{B_{(s)}}^{2} b^{2}\right]$,

where the constant $N_{B_{(s)}}$ is fixed by the normalization condition $\int \phi_{B_{(s)}}(x, b=0) d x=f_{B_{(s)}} /\left(2 \sqrt{2 N_{c}}\right)$ with the $B_{(s)}$ meson decay constant $f_{B_{(s)}}$, the shape parameter $\omega_{B_{(s)}}$ will be determined in the next section, and $b$ is the 
impact parameter conjugate to the transverse momentum $k_{T}$. It has been argued [38] that the contribution from $\bar{\phi}_{B}(k)$ is power suppressed, so it will be neglected in the numerical analysis below.

\section{B. SU(3) flavor structure}

To calculate hadronic two-body $B$ meson decay amplitudes systematically, we introduce the following SU(3) matrix elements for various species of mesons,

$$
\begin{aligned}
B^{-} & =(1,0,0), \quad \bar{B}=(0,1,0), \quad \bar{B}_{s}^{0}=(0,0,1), \\
M_{\pi^{+}} & =M_{\rho^{+}}=\left(\begin{array}{ccc}
0 & 0 & 0 \\
1 & 0 & 0 \\
0 & 0 & 0
\end{array}\right), \quad M_{K^{+}}=M_{K^{*+}}=\left(\begin{array}{lll}
0 & 0 & 0 \\
0 & 0 & 0 \\
1 & 0 & 0
\end{array}\right), \quad M_{K^{0}}=M_{K^{* 0}}=\left(\begin{array}{lll}
0 & 0 & 0 \\
0 & 0 & 0 \\
0 & 1 & 0
\end{array}\right), \\
\sqrt{2} M_{\pi^{0}} & =\sqrt{2} M_{\rho^{0}}=\left(\begin{array}{ccc}
1 & 0 & 0 \\
0 & -1 & 0 \\
0 & 0 & 0
\end{array}\right), \quad \sqrt{2} M_{\eta_{q}}=\sqrt{2} M_{\omega}=\left(\begin{array}{lll}
1 & 0 & 0 \\
0 & 1 & 0 \\
0 & 0 & 0
\end{array}\right), \quad M_{\eta_{s}}=M_{\phi}=\left(\begin{array}{lll}
0 & 0 & 0 \\
0 & 0 & 0 \\
0 & 0 & 1
\end{array}\right), \\
M_{\pi^{-}} & =M_{\rho^{-}}=M_{\pi^{+}}^{T}, \quad M_{K^{-}}=M_{K^{*-}}=M_{K^{+}}^{T}, \quad M_{\bar{K}^{0}}=M_{\bar{K}^{* 0}}=M_{K^{0}}^{T},
\end{aligned}
$$

which reflect the internal structure of the flavor SU(3) group. The isosinglet mesons like $\eta_{q}, \eta_{s}, \omega$, and $\phi$ will not be considered in the global analysis below, but their properties are listed here for completeness. We will take into account these hadrons, as extending the database in the future. The matrices relevant for the heavy-to-light transitions are given by

$$
\begin{gathered}
\delta_{u}=\left(\begin{array}{lll}
1 & 0 & 0 \\
0 & 0 & 0 \\
0 & 0 & 0
\end{array}\right), \quad \Lambda_{d}=\left(\begin{array}{l}
0 \\
1 \\
0
\end{array}\right), \\
\Lambda_{s}=\left(\begin{array}{l}
0 \\
0 \\
1
\end{array}\right), \quad e_{Q}=\left(\begin{array}{ccc}
1 & 0 & 0 \\
0 & -\frac{1}{2} & 0 \\
0 & 0 & -\frac{1}{2}
\end{array}\right) .
\end{gathered}
$$

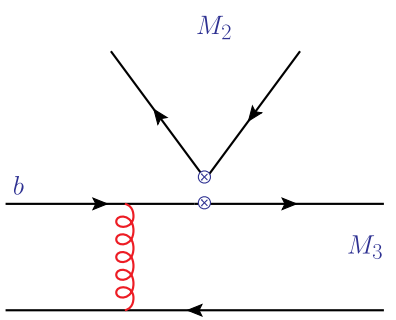

(a)

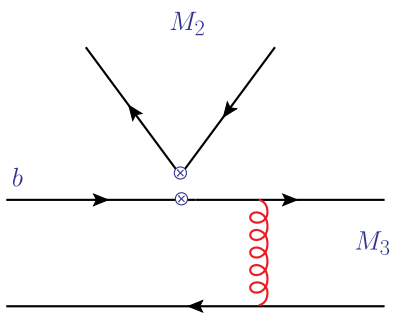

(b)

The factorization formula for a $B \rightarrow P P$ decay amplitude in Eq. (2) can be divided into four pieces, $F_{e}$ from the factorizable emission diagrams in Figs. 2(a) and 2(b), $M_{e}$ from the nonfactorizable emission diagrams in Figs. 2(c) and 2(d), $F_{a}$ from the factorizable annihilation diagrams in Figs. 3(a) and 3(b), and $M_{a}$ from the non-factorizable annihilation diagrams in Figs. 3(c) and 3(d), each of which contains at least one hard gluon exchange. All the diagrams receive contributions from the $(V-A)(V-A)$ operators denoted by $L L$, from the $(V-A)(V+A)$ operators denoted by $L R$, and from the $(S-P)(S+P)$ operators denoted by $S P$. The $(S-P)(S+P)$ operators appear under the Fierz transformation of the $(V-A)(V+A)$ ones. The explicit expressions for the above contributions, together with the Sudakov factors and hard kernels, are presented in the Appendix.

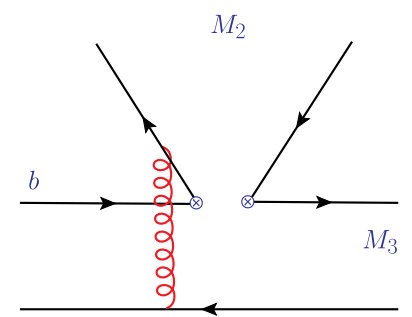

(c)

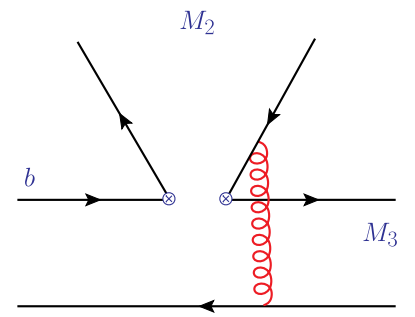

(d)

FIG. 2. Emission diagrams with possible four-quark operator insertions.

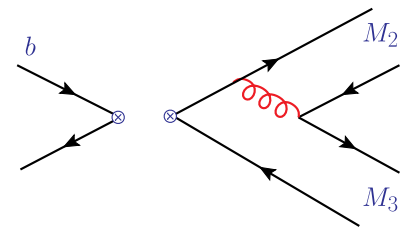

(a)

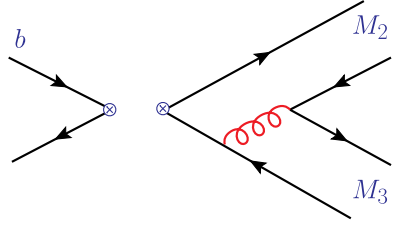

(b)

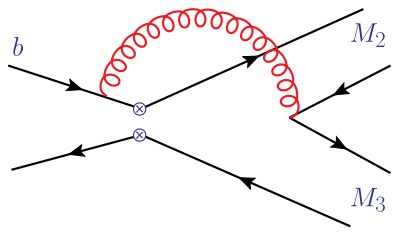

(c)

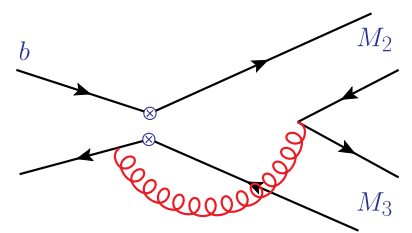

(d)

FIG. 3. Annihilation diagrams with possible four-quark operator insertions. 
We decompose the total $B \rightarrow M_{2} M_{3}$ decay amplitude into the combination

$$
\begin{aligned}
M= & \frac{G_{F}}{\sqrt{2}} V_{u b} V_{u q}^{*}\left[\mathcal{A}_{u}\left(B \rightarrow M_{2} M_{3}\right)\right] \\
& -\frac{G_{F}}{\sqrt{2}} V_{t b} V_{t q}^{*}\left[\mathcal{A}_{t}\left(B \rightarrow M_{2} M_{3}\right)\right],
\end{aligned}
$$

where $A_{u}\left(B \rightarrow M_{2} M_{3}\right)$ denotes the tree contribution with the product $V_{u b} V_{u f}^{*}$ of the CKM matrix elements, and $A_{t}\left(B \rightarrow M_{2} M_{3}\right)$ denotes the penguin contribution with the product $V_{t b} V_{t f}^{*}$. These amplitudes are written, in terms of the matrices in Eqs. (9) and (10), as

$$
\begin{aligned}
A_{u}\left(B \rightarrow M_{2} M_{3}\right)= & {\left[F_{e}^{L L}\left(a_{1}\right)+M_{e}^{L L}\left(C_{1}\right)\right] B M_{3} \delta_{u} M_{2} \Lambda_{f}+\left[F_{e}^{L L}\left(a_{2}\right)+M_{e}^{L L}\left(C_{2}\right)\right] B M_{3} \Lambda_{f} \operatorname{Tr}\left[\delta_{u} M_{2}\right] } \\
& +\left[F_{\text {ann }}^{L L}\left(a_{1}\right)+M_{\text {ann }}^{L L}\left(C_{1}\right)\right] B \delta_{u} M_{3} M_{2} \Lambda_{f}+\left[F_{\text {ann }}^{L L}\left(a_{2}\right)+M_{\text {ann }}^{L L}\left(C_{2}\right)\right] B \Lambda_{f} \operatorname{Tr}\left[\delta_{u} M_{3} M_{2}\right], \\
A_{t}\left(B \rightarrow M_{2} M_{3}\right)= & {\left[F_{e}^{L L}\left(a_{3}\right)+F_{e}^{L R}\left(a_{5}\right)+M_{e}^{L L}\left(C_{4}\right)+M_{e}^{S P}\left(C_{6}\right)\right] B M_{3} \Lambda_{f} \operatorname{Tr}\left[M_{2}\right] } \\
& +\left[F_{e}^{L L}\left(a_{4}\right)+F_{e}^{S P}\left(a_{6}\right)+M_{e}^{L L}\left(C_{3}\right)+M_{e}^{L R}\left(C_{5}\right)\right] B M_{3} M_{2} \Lambda_{f} \\
& +\left[F_{e}^{L R}\left(a_{7}\right)+F_{e}^{L L}\left(a_{9}\right)+M_{e}^{S P}\left(C_{8}\right)+M_{e}^{L L}\left(C_{10}\right)\right] B M_{3} \Lambda_{f} \operatorname{Tr}\left[e_{Q} M_{2}\right] \\
& +\left[F_{e}^{S P}\left(a_{8}\right)+F_{e}^{L L}\left(a_{10}\right)+M_{e}^{L R}\left(C_{7}\right)+M_{e}^{L L}\left(C_{9}\right)\right] B M_{3} e_{Q} M_{2} \Lambda_{f} \\
& +\left[F_{\text {ann }}^{L L}\left(a_{3}\right)+F_{\text {ann }}^{L R}\left(a_{5}\right)+M_{\text {ann }}^{L L}\left(C_{4}\right)+M_{\text {ann }}^{S P}\left(C_{6}\right)\right] B \Lambda_{f} \operatorname{Tr}\left[M_{3} M_{2}\right] \\
& +\left[F_{\text {ann }}^{L L}\left(a_{4}\right)+F_{\text {ann }}^{S P}\left(a_{6}\right)+M_{\text {ann }}^{L L}\left(C_{3}\right)+M_{\text {ann }}^{L R}\left(C_{5}\right)\right] B M_{3} M_{2} \Lambda_{f} \\
& +\left[F_{\text {ann }}^{L R}\left(a_{7}\right)+F_{\text {ann }}^{L L}\left(a_{9}\right)+M_{\text {ann }}^{S P}\left(C_{8}\right)+M_{\text {ann }}^{L L}\left(C_{10}\right)\right] B \Lambda_{f} \operatorname{Tr}\left[e_{Q} M_{3} M_{2}\right] \\
& +\left[F_{\text {ann }}^{S P}\left(a_{8}\right)+F_{\text {ann }}^{L L}\left(a_{10}\right)+M_{\text {ann }}^{L R}\left(C_{7}\right)+M_{\text {ann }}^{L L}\left(C_{9}\right)\right] B e_{Q} M_{3} M_{2} \Lambda_{f},
\end{aligned}
$$

with the Wilson coefficients $a_{1}=C_{2}+C_{1} / 3$, $a_{2}=C_{1}+C_{2} / 3, \quad a_{2 n-1}=C_{2 n-1}+C_{2 n} / 3, \quad$ and $a_{2 n}=$ $C_{2 n}+C_{2 n-1} / 3(n \geq 2)$. The unitarity of the CKM matrix is assumed in this work. The weak phase $\phi_{3}(\gamma)$ is defined via the CKM matrix element $V_{u b} \equiv\left|V_{u b}\right| e^{-i \gamma}$.

The $B \rightarrow V P$ decay amplitudes can be simply inferred from the $B \rightarrow P P$ amplitudes through the replacements of light meson LCDAs and of a chiral enhancement scale by a vector meson mass. For the $B \rightarrow V_{2} P_{3}$ emission and $B \rightarrow$ $P_{2} V_{3}$ annihilation, we apply the rule $\phi_{3}^{(2),(3)} \rightarrow-\phi_{3}^{(2),(3)}$, and further flip the signs of the $L R$ and $S P$ amplitudes, where the subscript (2) means twist 2, and (3) means twist 3 . For the $B \rightarrow V_{2} P_{3}$ annihilation, we apply $\phi_{3}^{(2)} \rightarrow-\phi_{3}^{(2)}$ and $\phi_{3}^{(3)} \rightarrow \phi_{3}^{(3)}$, and further flip the signs of the $L R$ and $S P$ amplitudes. The above rule holds for both the pseudoscalar $P$ and vector $V$ mesons, and for the factorizable and nonfactorizable diagrams. For the $B \rightarrow P_{2} V_{3}$ emission, we apply $\phi_{3}^{(2)} \rightarrow-\phi_{3}^{(2)}$ and $\phi_{3}^{(3)} \rightarrow \phi_{3}^{(3)}$, and further flip the signs of the nonfactorizable $L R$ amplitudes and the factorizable $S P$ amplitudes.

As shown in Eq. (5), there are nine Gegenbauer moments $a^{f} \mathrm{~S}$ in total for the twist-2 pseudoscalar LCDA $\phi_{P}(x)$ and twist-3 LCDAs $\phi_{P}^{P}(x)$ and $\phi_{P}^{T}(x)$. Note that the Gegenbauer moment $a_{1}^{\pi}$ vanishes due to the isospin symmetry, and $a_{P 4}^{f}$ are not included in the fit, because they cannot be constrained effectively under the current limited experimental accuracy. Thus, a $B \rightarrow P P$ decay amplitude contains $9 \times 9$ combinations of the Gegenbauer moments,

$$
M \sim \sum_{n, m=1}^{9} a_{n}^{f} a_{m}^{f} M_{n m}
$$

where the products $a_{n}^{f} a_{m}^{f}$ of the Gegenbauer moments $a_{2}^{\pi}$, $a_{4}^{\pi}, a_{P 2}^{\pi}, a_{T 2}^{\pi}, a_{2}^{\rho \|}, a_{1}^{K}, a_{2}^{K}, a_{4}^{K}$, and $a_{2}^{K^{*} \|}$ have been factored out explicitly. We compute the factorization formula $M_{n m}$, which involves only the Gegenbauer polynomials associated with $a_{n}^{f}$ and $a_{m}^{f}$, to establish a $9 \times 9$ database. Each database has 20 sets of values, corresponding to the Wilson coefficients $a_{1} \cdots a_{10}$ and $C_{1} \cdots C_{10}$ in Eq. (12). To analyze the $B \rightarrow V P$ decays, we construct a $9 \times 4 \times 2$ database for $M_{n m}$ in a similar manner, where $\times 2$ is attributed to the two possible final states $P_{2} V_{3}$ and $V_{2} P_{3}$. The inputs for the Fermi constant, the meson decay constants, the meson masses, and the chiral enhancement scale are the same as in [52], and the magnitudes of the CKM matrix elements are referred to [26] in the above computations.

\section{NUMERICAL RESULTS}

\section{A. Least-square fit and Bayesian analysis}

We determine the Gegenbauer moments and the weak phase $\phi_{3}(\gamma)$ by fitting the branching ratios and direct $C P$ asymmetries formulated from the decay amplitudes in Eq. (13) to experimental data using the nonlinear least$\chi^{2}$ method [53]. The least $-\chi^{2}$ method minimizes the summed residual $S$, 


$$
S=\sum_{i=1}^{n} r_{i}^{2}, \quad r_{i}=y_{i}-\hat{y}_{i}
$$

where $r_{i}$ is the residual at the $i$ th point $x_{i}, y_{i}\left(\hat{y}_{i}\right)$ represents the experimental data (response value), and $n$ is the number of data points. One defines a model function $\hat{y}_{i}=f\left(x_{i}, \vec{\beta}\right)$, where the vector $\vec{\beta}$ contains the $m$ adjustable parameters considered in the fit. The minimum of Eq. (14) is obtained by equating the gradient to zero,

$$
\frac{\partial S}{\partial \beta_{j}}=2 \sum_{i} r_{i} \frac{\partial f\left(x_{i}, \vec{\beta}\right)}{\partial \beta_{j}}=0, \quad j=1,2, \ldots, m .
$$

For a linear model, $f\left(x_{i}, \vec{\beta}\right)$ can be decomposed into a sum of multiple linear functions $G_{j}\left(x_{i}\right)$ with the corresponding coefficients $\beta_{j}, f\left(x_{i}, \vec{\beta}\right)=\sum_{j} \beta_{j} G_{j}\left(x_{i}\right)$. One then regards the function $G_{j}\left(x_{i}\right)$ as a matrix, and solves for $\beta_{j}$ from Eq. (15) with the data $y_{i}$.

A nonlinear model is more subtle, to which there is no closed-form solution in general. A possible approach is to select some initial values of the parameters, and refine the parameters by iteration. At each iteration, the model function $f\left(x_{i}, \vec{\beta}\right)$ is linearized through the first-order Taylor series expansion at $\beta_{j}^{k}$,

$$
\begin{aligned}
f\left(x_{i}, \vec{\beta}\right) & \approx f\left(x_{i}, \vec{\beta}^{k}\right)+\sum_{j} \frac{\partial f\left(x_{i}, \vec{\beta}\right)}{\partial \beta_{j}}\left(\beta_{j}-\beta_{j}^{k}\right), \\
& \equiv f\left(x_{i}, \vec{\beta}^{k}\right)+\sum_{j} J_{i j} \Delta \beta_{j},
\end{aligned}
$$

with $k$ being an iteration number, and $J$ being a Jacobian function. The minimum of the residual at this iteration is achieved by equating the gradient to zero,

$$
\frac{\partial S}{\partial \Delta \beta_{j}}=-2 \sum_{i=1}^{n} J_{i j}\left(y_{i}-f\left(x_{i}, \vec{\beta}^{k}\right)-\sum_{k=1}^{m} J_{i k} \Delta \beta_{k}\right)=0,
$$

where $\Delta \beta_{j}$ can be solved by inverting the Jacobian matrix. The parameters then take the values $\beta_{j}^{k+1}=\beta_{j}^{k}+\Delta \beta_{j}$ for the next iteration.

Because of the approximations in the Taylor expansion and in the matrix inversion (if the Jacobian matrix is not a square one), no algorithm works for all nonlinear models, and fit results may be sensitive to initial conditions. To stabilize a complicated nonlinear fit, one can perform a Bayesian analysis (conditionally biased fit). Instead of the single $\chi^{2}$ term corresponding to Eq. (14),

$$
\chi^{2}=\sum_{i=1}^{n}\left(\frac{y_{i}-\hat{y}_{i}}{\delta y_{i}}\right)^{2}
$$

with $\delta y_{i}$ being the errors of experimental data, we employ a modified version

$$
\chi_{m}^{2}=\chi^{2}+\chi_{\text {prior }}^{2}, \quad \chi_{\text {prior }}^{2}=\sum_{j} \frac{\left(\beta_{j}-\tilde{\beta}_{j}\right)^{2}}{\tilde{\sigma}_{j}^{2}} .
$$

The second term $\chi_{\text {prior }}^{2}$ is a stabilizing function, where $\tilde{\beta}_{j}$ are artificially introduced default values for the fitted parameters with some errors $\tilde{\sigma}_{j}$. When the summed residual in Eq. (19) are minimized, the $\chi_{\text {prior }}^{2}$ term favors $\beta_{j}$ in the range $\left(\tilde{\beta}_{j}-\tilde{\sigma}_{j}, \tilde{\beta}_{j}+\tilde{\sigma}_{j}\right)$. The values of $\tilde{\beta}_{j}$ and $\tilde{\sigma}_{j}$ should be chosen reasonably according to prior physical knowledge on the fitted parameters. In the present study we select the Gegenbauer moments derived in QCD sum rules as our Bayesian data $\tilde{\beta}$, and five times of the errors from QCD sum rules for our $\tilde{\sigma}$, which reduce the weight of $\chi_{\text {prior }}^{2}$ in the fit.

The Bayesian analysis is one of the standard methods for data fitting, which has been widely adopted in data analysis for high-energy experimental physics and lattice calculations. We repeat that the introduction of the $\chi_{\text {prior }}^{2}$ term in Eq. (19) is to make fitted results, especially those in complicated processes like hadronic $B$ meson decays, more stable. It is very difficult to fit the Gegenbauer moments of higher orders or in higher-twist LCDAs. Applying the Bayesian analysis, we can stabilize our fits and exclude results in unfavored regions, such as the regions with Gegenbauer moments much larger than unity. Although the sum-rule values have been input as the prior information for the analysis, the associated uncertainties are magnified by five times to reduce the bias effect to a very low level. To verify this statement, we compare the fitted leading moment $a_{2}^{\pi}$ in the Bayesian and unbiased fits. The former yields $a_{2}^{\pi}=0.644 \pm 0.075$ as shown in Table I, while the latter returns $a_{2}^{\pi}=0.635 \pm 0.098$, which are consistent with each other within errors, and differ from the sumrule input $a_{2}^{\pi}=0.25 \pm 0.15$ also given in Table I. This comparison confirms that the bias effect is indeed minor in our fits.

\section{B. $B_{s}$ meson distribution amplitude}

We point out that the $B_{(s)}$ meson DA appears in all the $B_{(s)} \rightarrow M_{2} M_{3}$ decay amplitudes, so it is difficult to extract the shape parameters $\omega_{B_{(s)}}$ in a global fit. The investigation in [38] shows that $\omega_{B}=0.4 \mathrm{GeV}$ for the $B$ meson DA leads to reasonable results for the $B \rightarrow \pi$ transition form factors, which agree with those from light cone sum rules and lattice QCD. Therefore, we choose this value as the input, and perform the global fit to determine the Gegenbauer moments of the light meson LCDAs. As to the shape parameter $\omega_{B_{s}}$ in the $B_{s}$ meson DA, it is observed that the Gegenbauer moments fitted from the $B_{s} \rightarrow P P$ data are not sensitive to its variation: we scan the range of $\omega_{B_{s}}$ from 0.4 to $0.6 \mathrm{GeV}$, and make sure that the fitted Gegenbauer 
TABLE I. Gegenbauer moments and the $\gamma$ angle from a joint fit for the twist- 2 and twist-3 LCDAs. The Gegenbauer moments from QCD sum rules (QSR) [5-7], lattice QCD (LQCD) [55,56] and the light-front quark model (LF) [57] are also listed for comparison.

\begin{tabular}{lccccccc}
\hline \hline & $a_{1}^{\pi}$ & $a_{2}^{\pi}$ & $a_{4}^{\pi}$ & $a_{P 2}^{\pi}$ & $a_{T 2}^{\pi}$ & $a_{1}^{\rho \|}$ & $a_{2}^{\rho \|}$ \\
\hline Fit & $\ldots$ & $0.644 \pm 0.075$ & $-0.41 \pm 0.098$ & $1.08 \pm 0.15$ & $-0.48 \pm 0.33$ & $\ldots$ & $0.16 \pm 0.084$ \\
QSR & $\ldots$ & $0.25 \pm 0.15$ & $-0.015 \pm 0.025$ & $\ldots$ & $\ldots$ & $\ldots$ & $0.15 \pm 0.07$ \\
LQCD & $\ldots$ & $0.101 \pm 0.024$ & $\ldots$ & $\ldots$ & $\ldots$ & $\ldots$ & $0.132 \pm 0.0027$ \\
LF & $\ldots$ & $0.12 \pm 0.05$ & $-0.003 \pm 0.03$ & $\ldots$ & $\ldots$ & $\ldots$ & $0.02 \pm 0.02$ \\
& $a_{1}^{K}$ & $a_{2}^{K}$ & $a_{4}^{K}$ & $a_{P 2}^{K}$ & $a_{T 2}^{K}$ & $a_{1}^{K^{*} \|}$ & $a_{2}^{K^{*} \|}$ \\
\hline Fit & $0.331 \pm 0.082$ & $0.28 \pm 0.10$ & $-0.398 \pm 0.073$ & $\ldots$ & $\ldots$ & $\ldots$ & $0.137 \pm 0.029$ \\
QSR & $0.06 \pm 0.03$ & $0.25 \pm 0.15$ & $\ldots$ & $\ldots$ & $\ldots$ & $0.03 \pm 0.02$ & $0.11 \pm 0.09$ \\
LQCD & $0.053 \pm 0.003$ & $0.09 \pm 0.02$ & $\ldots$ & $\ldots$ & $\ldots$ & $\ldots$ & $\cdots$ \\
LF & $0.09 \pm 0.13$ & $0.03 \pm 0.03$ & $0.02 \pm 0.03$ & $\ldots$ & $\ldots$ & $0.11 \pm 0.14$ & $-0.03 \pm 0.07$ \\
\hline \hline
\end{tabular}

moments are relatively stable in the range $0.45 \mathrm{GeV}<$ $\omega_{B_{s}}<0.55 \mathrm{GeV}$. We then select the data of several precisely measured channels, $B_{s} \rightarrow K^{+} K^{-}, K^{+} \pi^{-}, K^{0} \bar{K}^{0}$, and $\pi^{+} \pi^{-}$, and compare them with the reconstructed data from the PQCD factorization formulas. The comparison displayed in Fig. 4, where the red bands are from the experimental data and the blue bands are from the reconstructed data, indicates that the choice $\omega_{B_{s}}=0.48 \mathrm{GeV}$ is preferred: with this value of $\omega_{B_{s}}$, the PQCD results from
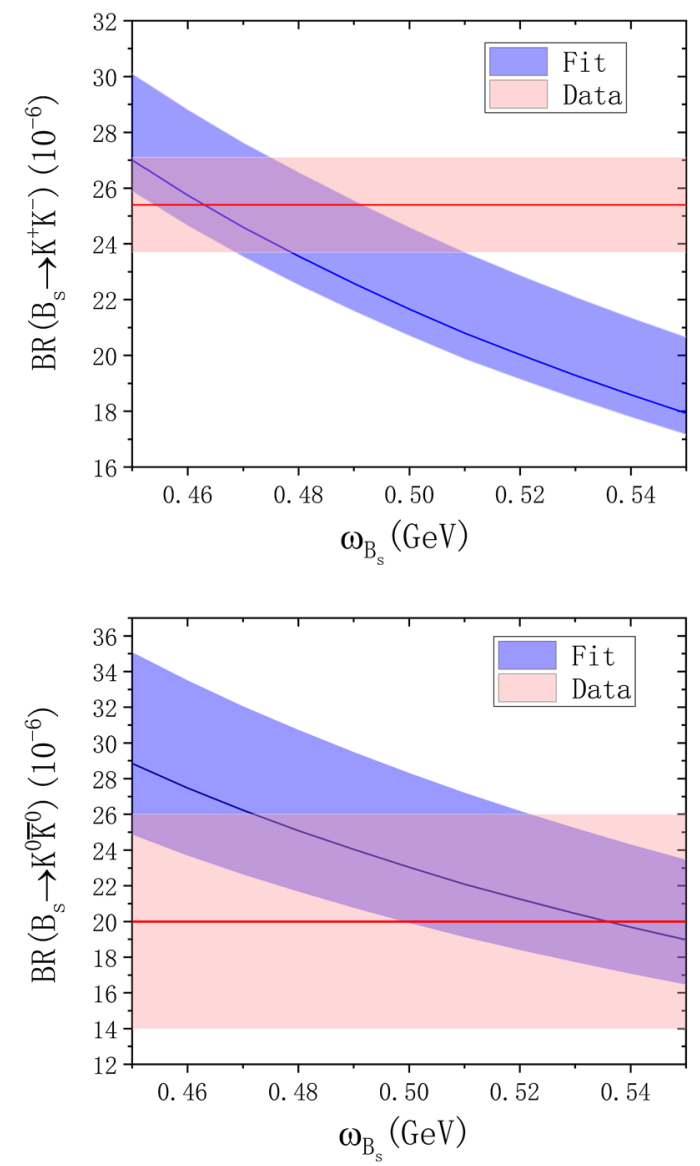

the fit accommodate the selected four piece of data simultaneously.

\section{Global fit}

We fit the PQCD factorization formulas with the database constructed in the previous section to the measured branching ratios and direct $C P$ asymmetries $A_{C P}$ in the $B_{(s)} \rightarrow P P, V P$ decays, which are collected in the left columns of Table II. The $A_{C P}$ data marked in red, which
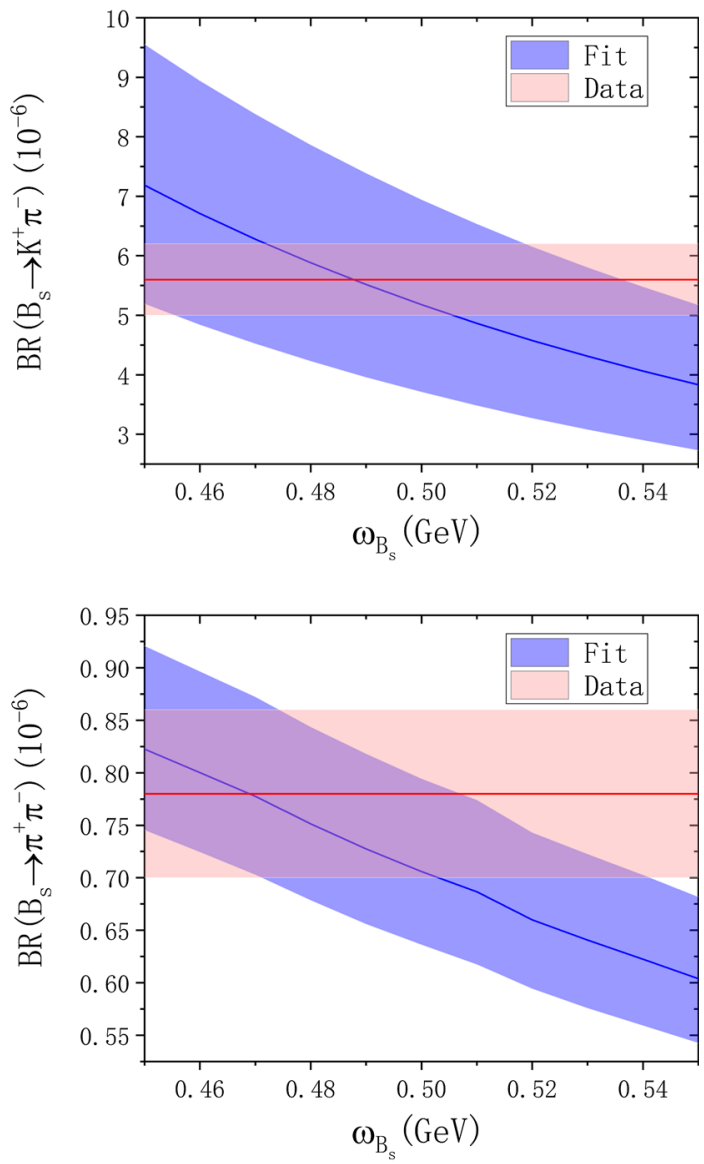

FIG. 4. Dependencies of the experimental data and the reconstructed data on $\omega_{B_{s}}$. 
TABLE II. Experimental data for branching ratios and direct $C P$ asymmetries $A_{C P}$ [26], and the theoretical results derived from the fitted Gegenbauer moments in Table I. The data with precision less than $3 \sigma$ are marked in red.

\begin{tabular}{|c|c|c|c|c|}
\hline \multirow[b]{2}{*}{ Channel } & \multicolumn{2}{|c|}{ Data } & \multicolumn{2}{|c|}{ Fit } \\
\hline & Branching ratio $\left(10^{-6}\right)$ & $A_{C P}(\%)$ & Branching ratio $\left(10^{-6}\right)$ & $A_{C P}(\%)$ \\
\hline$B^{0} \rightarrow \bar{K}^{0} K^{0}$ & $1.21 \pm 0.16$ & $-60 \pm 70$ & $1.23 \pm 0.08$ & $0 \pm 0$ \\
\hline$B^{0} \rightarrow \bar{K}^{0} \pi^{0}$ & $9.90 \pm 0.50$ & $0 \pm 13$ & $8.98 \pm 0.19$ & $-4.02 \pm 0.48$ \\
\hline$B^{0} \rightarrow K^{-} \pi^{+}$ & $19.6 \pm 0.50$ & $-8.3 \pm 0.6$ & $20.3 \pm 0.36$ & $-8.34 \pm 0.36$ \\
\hline$B^{0} \rightarrow \pi^{-} \pi^{+}$ & $5.12 \pm 0.19$ & $32 \pm 4$ & $5.24 \pm 0.17$ & $23.2 \pm 2.1$ \\
\hline$B^{0} \rightarrow \rho^{0} \bar{K}^{0}$ & $3.40 \pm 1.10$ & $4 \pm 20$ & $3.06 \pm 0.37$ & $2.853 \pm 0.068$ \\
\hline$B^{0} \rightarrow \pi^{0} \bar{K}^{* 0}$ & $3.30 \pm 0.60$ & $-15 \pm 13$ & $1.73 \pm 0.10$ & $-6.02 \pm 0.6$ \\
\hline$B^{0} \rightarrow \pi^{-} \rho^{+} / \pi^{+} \rho^{-}$ & $23.0 \pm 2.30$ & $13 \pm 6 /-8 \pm 8$ & $23.33 \pm 0.8$ & $-24.3 \pm 1 / 8.1 \pm 1.1$ \\
\hline$B^{-} \rightarrow K^{0} K^{-}$ & $1.31 \pm 0.17$ & $4 \pm 14$ & $1.47 \pm 0.09$ & $22.5 \pm 2.7$ \\
\hline$B^{-} \rightarrow \pi^{0} K^{-}$ & $12.9 \pm 0.50$ & $3.7 \pm 2.1$ & $12.99 \pm 0.23$ & $-6.44 \pm 0.6$ \\
\hline$B^{-} \rightarrow \bar{K}^{0} \pi^{-}$ & $23.7 \pm 0.80$ & $-1.7 \pm 1.6$ & $23.15 \pm 0.42$ & $-2.84 \pm 0.24$ \\
\hline$B^{-} \rightarrow \rho^{-} \pi^{0}$ & $10.9 \pm 1.40$ & $2 \pm 11$ & $8.73 \pm 0.25$ & $24.2 \pm 2.3$ \\
\hline$B^{-} \rightarrow \pi^{0} K^{*-}$ & $6.80 \pm 0.90$ & $-39 \pm 21$ & $3.51 \pm 0.19$ & $-33.5 \pm 1.7$ \\
\hline$B^{-} \rightarrow K^{-} K^{* 0}$ & $0.59 \pm 0.08$ & $12 \pm 10$ & $0.476 \pm 0.022$ & $22.5 \pm 1.3$ \\
\hline$B_{s} \rightarrow K^{-} K^{+}$ & $26.6 \pm 2.20$ & $-14 \pm 11$ & $24.8 \pm 1.50$ & $-8.1 \pm 2.3$ \\
\hline$B_{s} \rightarrow \pi^{-} \pi^{+}$ & $0.7 \pm 0.1$ & $\ldots$ & $0.798 \pm 0.092$ & $-1.62 \pm 0.39$ \\
\hline$B_{s} \rightarrow K^{0} \bar{K}^{0}$ & $20.0 \pm 6.00$ & $0 \pm 0$ & $26.2 \pm 1.60$ & $0 \pm 0$ \\
\hline$B_{s} \rightarrow \pi^{-} K^{+}$ & $5.80 \pm 0.70$ & $22.1 \pm 1.5$ & $5.69 \pm 0.64$ & $22.1 \pm 1.2$ \\
\hline$B_{s} \rightarrow K^{+} K^{*-} / K^{-} K^{*+}$ & $19.0 \pm 5.0$ & $\ldots$ & $15.28 \pm 0.90$ & $-33.8 \pm 1.3 / 53.5 \pm 2.4$ \\
\hline$B_{s} \rightarrow K^{0} \bar{K}^{* 0} / \bar{K}^{0} K^{* 0}$ & $20.0 \pm 6.00$ & $\cdots$ & $15.06 \pm 0.96$ & $0 \pm 0$ \\
\hline
\end{tabular}

have larger errors, do not provide a strong constraint in the fit. Note that the LHCb Collaboration has updated their prediction for $A_{C P}\left(B^{0} \rightarrow \bar{K}^{0} \pi^{0}\right)$, which reads $(-13.8 \pm$ $2.5) \%$ [57], based on the $A_{C P}\left(B^{+} \rightarrow K^{+} \pi^{0}\right)$ measurement via the sum rule for these direct $C P$ asymmetries. The data of those modes, which are greatly affected by subleading contributions according to the existent PQCD calculations $[52,58,59]$, namely, suffer significant theoretical uncertainties, are excluded in our fit. The Gegenbauer moments of both the twist-2 and twist-3 LCDAs from a joint fit, corresponding to the shape parameters $w_{B}=0.4 \mathrm{GeV}$ and $w_{B_{s}}=0.48 \mathrm{GeV}$, have been collected in Tables I with $\chi^{2} /$ d.o.f. $=0.77$. These values represent the Gegenbauer moments at the scale of $1 \mathrm{GeV}$, where the LCDAs are defined [58]. The errors in our fit mainly arise from the experimental uncertainty. The $\chi_{\text {prior }}^{2}$ term in the Bayesian analysis introduces little error to the fit results. Some higher-order moments or moments of higher-twist LCDAs cannot be constrained effectively due to the current limited experimental accuracy. This is the reason why the values of the moments $a_{P 2}^{K}, a_{T 2}^{K}$, and $a_{1}^{K * \|}$ are not presented in Table I. Overall speaking, our results are closer to those from QCD sum rules than to those from lattice QCD $[54,55]$ and the light-front quark model [56].

It is seen that some fitted Gegenbauer moments, like $a_{2}^{\rho \|}$, $a_{2}^{K}$, and $a_{2}^{K^{*} \|}$, agree well with those from QCD sum rules $[5,7]$ within $1 \sigma$ error, which are listed in Table I for comparison. We stress that our fit is based on the LO PQCD factorization formulas, and that next-to-leading-order
(NLO) corrections change the heavy-to-light transition form factors by about $30 \%$ [60,61]. It is difficult to estimate how much systematic error is caused by NLO effects for the fitting at LO, because NLO corrections to the nonfactorizable amplitudes in hadronic two-body $B$ meson decays have not yet been completed in the PQCD approach. Higherpower contributions (such as the power-suppressed contribution from another $B$ meson DA $\bar{\phi}_{B}$ in Eq. (8), which was shown to be of the same order as the NLO one in [62]) have not been taken into account either. Therefore, it is likely that some fitted Gegenbauer moments, like $a_{2}^{\pi}$, differ more significantly from those in QCD sum rules. The outcome of $a_{P 2}^{\pi}$, slightly larger than unity, can be reduced by including the higher moment $a_{P 4}^{\pi}$ into the fit. As explained before, $a_{P 4}^{\pi}$ is not considered here, because it cannot be constrained effectively under the current experimental accuracy. It is worth mentioning that the weak phase $\phi_{3}(\gamma)$ is found to be $(75.2 \pm 2.9)^{\circ}$, consistent with the value $\left(72.1_{-4.5}^{+4.1}\right)^{\circ}$ in Particle Data Group [26], and $(69.8 \pm 2.1 \pm 0.9)^{\circ}$ from the factorization-assisted topological diagram approach [63]. The agreements of our results with the Gegenbauer moments from sum rules and with $\phi_{3}(\gamma)$ extracted in other methods support the PQCD factorization for hadronic twobody $B$ meson decays.

With the fitted Gegenbauer moments in Table I, we calculate the branching ratios and $A_{C P}$ in the LO PQCD approach, and present the results in the right columns of Table II. It is observed that all the considered data, except the $B^{-} \rightarrow \pi^{0} K^{*-}$ branching ratio, are well reproduced. The observables removed from the fit, i.e., those suffering 
TABLE III. LO PQCD predictions for the observables removed from the fit, and compared with those in previous PQCD analyses $[52,58,59,66]$.

\begin{tabular}{|c|c|c|c|c|c|}
\hline \multirow[b]{2}{*}{ Channel } & \multicolumn{2}{|c|}{ Data } & \multicolumn{2}{|l|}{ Fit } & \multirow{2}{*}{$\begin{array}{c}\text { PQCD } \\
\text { Branching ratio }\end{array}$} \\
\hline & Branching ratio $\left(10^{-6}\right)$ & $A_{C P}(\%)$ & Branching ratio $\left(10^{-6}\right)$ & $A_{C P}(\%)$ & \\
\hline$B^{0} \rightarrow K^{+} K^{-}$ & $0.078 \pm 0.015$ & $\ldots$ & $0.155 \pm 0.027$ & $52.0 \pm 15.0$ & \\
\hline$B^{0} \rightarrow \pi^{+} K^{*-}$ & $7.5 \pm 0.4$ & $-27 \pm 4$ & $4.93 \pm 0.28$ & $-52.0 \pm 2.1$ & $5.1[58]$ \\
\hline$B^{0} \rightarrow \pi^{0} \rho^{0}$ & $2.0 \pm 0.5$ & $-27 \pm 24$ & $0.026 \pm 0.0022$ & $-47 \pm 21$ & $0.15[59]$ \\
\hline$B^{0} \rightarrow K^{-} \rho^{+}$ & $7.0 \pm 0.9$ & $20 \pm 11$ & $4.41 \pm 0.6$ & $48.3 \pm 4.9$ & $4.7[58]$ \\
\hline$B^{-} \rightarrow \rho^{-} \bar{K}^{0}$ & $7.3 \pm 1.2$ & $-3 \pm 15$ & $3.39 \pm 0.55$ & $3.18 \pm 0.55$ & $3.6[58]$ \\
\hline$B^{-} \rightarrow \rho^{0} K^{-}$ & $3.7 \pm 0.5$ & $37 \pm 1$ & $2.24 \pm 0.41$ & $69.7 \pm 3.0$ & $2.5[58]$ \\
\hline$B^{-} \rightarrow \pi^{-} \bar{K}^{* 0}$ & $10.1 \pm 0.8$ & $-4 \pm 9$ & $5.17 \pm 0.23$ & $-0.61 \pm 0.19$ & $5.5[58]$ \\
\hline$B^{-} \rightarrow \pi^{-} \rho^{0}$ & $8.3 \pm 1.2$ & $0.009 \pm 0.019$ & $4.61 \pm 0.36$ & $-35.3 \pm 1.8$ & $\sim 5.39[66]$ \\
\hline$B_{s} \rightarrow \pi^{-} K^{*+}$ & $2.9 \pm 1.1$ & $\ldots$ & $9.53 \pm 0.24$ & $-25.5 \pm 1.0$ & $7.6[52]$ \\
\hline
\end{tabular}

significant theoretical uncertainties, are also predicted in the LO PQCD formalism, and compared with the data in Table III. The predicted branching ratios are very close to the values obtained in the previous PQCD calculations, so the deviation from the data remains. In particular, $A_{C P}$ in the $B^{-} \rightarrow \pi^{-} \rho^{0}$ mode has been predicted to be large and negative in most QCD approaches $[64,65]$, but its data are as small as $0.009 \pm 0.019$ [26]. The inclusion of higherorder and higher-power contributions to hadronic two-body $B$ meson decays may improve the consistency. The theoretical errors given in Tables II and III arise only from those of the fitted Gegenbauer moments and $\phi_{3}(\gamma)$. More precise measurements are urged, and subleading contributions should be included into the PQCD framework to strengthen the constraint on the Gegenbauer moments and to sharpen the confrontation between theoretical predictions and experimental data.

\section{Remarks and future developments}

A few remarks are given as follows:

(i) We have focused only on the branching ratios and direct $C P$ asymmetries in the $B \rightarrow P P, V P$ decays, and neglected those modes involving isosinglet mesons in the above analysis. Other observables, such as mixing-induced $C P$ asymmetries and polarizations in $B \rightarrow V V$ decays, can be included straightforwardly. Though more parameters will be introduced through LCDAs for transversely polarized vector mesons, sufficient precise measurements on polarization observables can be achieved at $\mathrm{LHCb}$ and Belle-II.

(ii) LCDAs also appear in the factorization formulas for heavy-to-light transition form factors that govern semileptonic $B$ meson decays. One can take into account experimental constraints from these decays in the future, in particular those from their dependence on the lepton-pair invariant mass squared $q^{2}$.

(iii) As we have pointed out, decay widths of a few modes are suppressed at LO in PQCD, and may be well described with the inclusion of higher-order contributions $[58,67]$. Some sources of power corrections have been explored in Refs. [68,69]. A new database will be established in a similar way by using the flavor structure for these radiative and power corrections, via which the precision of a global analysis can be enhanced.

(iv) We did not consider all systematic and parametric uncertainties in the current analysis, such as the ones originating from the variations of factorization scales and nonperturbative QCD parameters.

(v) If a high-precision global study reveals notable tensions between theoretical results and experimental data in the future, it may hint that new physics effects are inevitable. One is then motivated to include new physics contributions, which can also be analyzed according to the flavor structure of new physics operators.

\section{SUMMARY}

As stated in the introduction, nonperturbative hadron LCDAs provide one of major sources of theoretical uncertainties in all the factorization-based approaches to hadronic two-body $B$ meson decays. In this paper we have performed a global analysis of the light meson LCDAs by fitting the LO PQCD factorization formulas for $B_{(s)} \rightarrow$ $P P, V P$ decays to available data of branching ratios and direct $C P$ asymmetries. A computation code was developed based on the flavor structure of the four-quark effective operators to establish the database, which contains the part of decay amplitudes without the Gegenbauer moments. This database facilitates the global fit, from which the Gegenbauer moments of the twist-2 and twist-3 LCDAs for the pseudoscalar meson $P(P=\pi, K)$ and vector meson $V$ ( $V=\rho, K^{*}$ ) were determined. Most of our fit results agree with the moments derived in QCD sum rules, and those with discrepancies deserve more thorough investigation that takes into account higher-order and higher-power corrections in the PQCD approach. The weak phase 
$\phi_{3}(\gamma)=(75.2 \pm 2.9)^{\circ}$ in consistency with the value in Particle Data Group was also extracted. Predictions for the modes, which were excluded in the fit due to large theoretical uncertainties, are close to the existent PQCD results, and still deviate from the data. To improve the consistency, subleading contributions to hadronic two-body $B$ meson decays need to be included, when their derivation is completed in the future.

Since the $B_{(s)}$ meson DA appears in all the $B_{(s)} \rightarrow M_{2} M_{3}$ decay amplitudes, it is difficult to constrain the shape parameters $\omega_{B_{(s)}}$ in this DA in a global fit. The shape parameter $\omega_{B}=0.4 \mathrm{GeV}$ is an input, and $\omega_{B_{s}}=0.48 \mathrm{GeV}$ is subject to a discretionary choice in the present study. The difficulty is expected to be overcome, when data for exclusive processes other than hadronic two-body $B$ meson decays are considered in the global fit. This is a straightforward extension of the framework proposed here, through which the global determination of LCDAs for other hadrons is also feasible. We have initiated a program for exclusive processes parallel to global fits of parton distribution functions for inclusive processes. It is worth a further pursuit with long-term and large-scale endeavor.

\section{ACKNOWLEDGMENTS}

W. W. thanks Professor Deshan Yang for a long discussion on a possible global analysis under the flavor SU(3) structure in the PQCD approach. J. H. thanks Dr. Dacheng Yan for useful discussions on two-body $B$ meson decays. J. H. is supported by NSFC under Grant No. 11947215 . J. H., W. W., and Z.P.X. are supported in part by Natural Science Foundation of China under Grants No. 11735010 and No. U2032102, and by Natural Science Foundation of Shanghai under Grant No. 15DZ2272100. H. N. L. is supported by MOST of R.O.C. under Grant No. MOST-1072119-M-001-035-MY3. C. D. L. is supported by National Science Foundation of China under Grants No. 11521505 and No. 12070131001. The computation package to construct the database in this work is available on request.

\section{APPENDIX: $B \rightarrow P P$ DECAY AMPLITUDES}

The explicit LO PQCD factorization formulas for the $B \rightarrow P P$ decay amplitudes from the various current operators and topologies are presented in this appendix, with $C_{F}=4 / 3$, the Wilson coefficients $a_{i}$, and $r_{i}=m_{0 i} / m_{B}$, where $m_{0 i}$ is the chiral enhancement scale:

$$
\begin{aligned}
& F_{e}^{L L}\left(a_{i}\right)=8 \pi C_{F} m_{B}^{4} f_{P_{2}} \int_{0}^{1} d x_{1} d x_{3} \int_{0}^{\infty} b_{1} d b_{1} b_{3} d b_{3} \phi_{B}\left(x_{1}, b_{1}\right)\left\{a_{i}\left(t_{a}\right) E_{e}\left(t_{a}\right)\right. \\
& \times\left[\left(2-x_{3}\right) \phi_{3}^{A}\left(x_{3}\right)+r_{3}\left(2 x_{3}-1\right)\left(\phi_{3}^{P}\left(x_{3}\right)-\phi_{3}^{T}\left(x_{3}\right)\right)\right] h_{e}\left(x_{1}, 1-x_{3}, b_{1}, b_{3}\right) \\
& \left.+2 r_{3} \phi_{3}^{P}\left(x_{3}\right) a_{i}\left(t_{a}^{\prime}\right) E_{e}\left(t_{a}^{\prime}\right) h_{e}\left(1-x_{3}, x_{1}, b_{3}, b_{1}\right)\right\} \text {, } \\
& F_{e}^{L R}\left(a_{i}\right)=-F_{e}^{L L}\left(a_{i}\right), \\
& F_{e}^{S P}\left(a_{i}\right)=16 \pi r_{2} C_{F} m_{B}^{4} f_{M_{2}} \int_{0}^{1} d x_{1} d x_{3} \int_{0}^{\infty} b_{1} d b_{1} b_{3} d b_{3} \phi_{B}\left(x_{1}, b_{1}\right)\left\{a_{i}\left(t_{a}\right) E_{e}\left(t_{a}\right)\right. \\
& \times\left[\phi_{3}^{A}\left(x_{3}\right)+r_{3}\left(3-x_{3}\right) \phi_{3}^{P}\left(x_{3}\right)+r_{3}\left(1-x_{3}\right) \phi_{3}^{T}\left(x_{3}\right)\right] h_{e}\left(x_{1}, 1-x_{3}, b_{1}, b_{3}\right) \\
& \left.+2 r_{3} \phi_{3}^{P}\left(x_{3}\right) a_{i}\left(t_{a}^{\prime}\right) E_{e}\left(t_{a}^{\prime}\right) h_{e}\left(1-x_{3}, x_{1}, b_{3}, b_{1}\right)\right\}, \\
& M_{e}^{L L}\left(C_{i}\right)=32 \pi C_{F} m_{B}^{4} / \sqrt{6} \int_{0}^{1} d x_{1} d x_{2} d x_{3} \int_{0}^{\infty} b_{1} d b_{1} b_{2} d b_{2} \phi_{B}\left(x_{1}, b_{1}\right) \phi_{2}^{A}\left(x_{2}\right) \\
& \times\left\{\left[x_{2} \phi_{3}^{A}\left(x_{3}\right)+r_{3}\left(x_{3}-1\right)\left(\phi_{3}^{P}\left(x_{3}\right)+\phi_{3}^{T}\left(x_{3}\right)\right)\right] C_{i}\left(t_{b}\right) E_{e}^{\prime}\left(t_{b}\right)\right. \\
& \times h_{n}\left(x_{1}, x_{2}, 1-x_{3}, b_{1}, b_{2}\right)+h_{n}\left(x_{1}, 1-x_{2}, 1-x_{3}, b_{1}, b_{2}\right) \\
& \left.\times\left[-\left(2-x_{2}-x_{3}\right) \phi_{3}^{A}\left(x_{3}\right)+r_{3}\left(1-x_{3}\right)\left(\phi_{3}^{P}\left(x_{3}\right)-\phi_{3}^{T}\left(x_{3}\right)\right)\right] C_{i}\left(t_{b}^{\prime}\right) E_{e}^{\prime}\left(t_{b}^{\prime}\right)\right\} \text {, } \\
& M_{e}^{L R}\left(C_{i}\right)=32 \pi C_{F} m_{B}^{4} r_{2} / \sqrt{6} \int_{0}^{1} d x_{1} d x_{2} d x_{3} \int_{0}^{\infty} b_{1} d b_{1} b_{2} d b_{2} \phi_{B}\left(x_{1}, b_{1}\right) \\
& \times\left\{h _ { n } ( x _ { 1 } , x _ { 2 } , 1 - x _ { 3 } , b _ { 1 } , b _ { 2 } ) \left[r_{3}\left(1-x_{3}\right)\left(\phi_{2}^{P}\left(x_{2}\right)+\phi_{2}^{T}\left(x_{2}\right)\right)\left(\phi_{3}^{P}\left(x_{3}\right)-\phi_{3}^{T}\left(x_{3}\right)\right)\right.\right. \\
& \left.+r_{3} x_{2}\left(\phi_{2}^{P}\left(x_{2}\right)-\phi_{2}^{T}\left(x_{2}\right)\right)\left(\phi_{3}^{P}\left(x_{3}\right)+\phi_{3}^{T}\left(x_{3}\right)\right)+x_{2} \phi_{3}^{A}\left(x_{3}\right)\left(\phi_{2}^{P}\left(x_{2}\right)-\phi_{2}^{T}\left(x_{2}\right)\right)\right] C_{i}\left(t_{b}\right) E_{e}^{\prime}\left(t_{b}\right) \\
& +h_{n}\left(x_{1}, 1-x_{2}, 1-x_{3}, b_{1}, b_{2}\right)\left[\left(x_{2}-1\right) \phi_{3}^{A}\left(x_{3}\right)\left(\phi_{2}^{P}\left(x_{2}\right)+\phi_{2}^{T}\left(x_{2}\right)\right)\right. \\
& +r_{3}\left(x_{2}-1\right)\left(\phi_{2}^{P}\left(x_{2}\right)+\phi_{2}^{T}\left(x_{2}\right)\right)\left(\phi_{3}^{P}\left(x_{3}\right)+\phi_{3}^{T}\left(x_{3}\right)\right) \\
& \left.\left.+r_{3}\left(x_{3}-1\right)\left(\phi_{2}^{P}\left(x_{2}\right)-\phi_{2}^{T}\left(x_{2}\right)\right)\left(\phi_{3}^{P}\left(x_{3}\right)-\phi_{3}^{T}\left(x_{3}\right)\right)\right] C_{i}\left(t_{b}^{\prime}\right) E_{e}^{\prime}\left(t_{b}^{\prime}\right)\right\},
\end{aligned}
$$




$$
\begin{aligned}
M_{e}^{S P}\left(C_{i}\right)= & 32 \pi C_{F} m_{B}^{4} / \sqrt{6} \int_{0}^{1} d x_{1} d x_{2} d x_{3} \int_{0}^{\infty} b_{1} d b_{1} b_{2} d b_{2} \phi_{B}\left(x_{1}, b_{1}\right) \phi_{2}^{A}\left(x_{2}\right) \\
& \times\left\{\left[\left(x_{3}-1-x_{2}\right) \phi_{3}^{A}\left(x_{3}\right)+r_{3}\left(1-x_{3}\right)\left(\phi_{3}^{P}\left(x_{3}\right)-\phi_{3}^{T}\left(x_{3}\right)\right)\right]\right. \\
& \times C_{i}\left(t_{b}\right) E_{e}^{\prime}\left(t_{b}\right) h_{n}\left(x_{1}, x_{2}, 1-x_{3}, b_{1}, b_{2}\right)+C_{i}\left(t_{b}^{\prime}\right) E_{e}^{\prime}\left(t_{b}^{\prime}\right) \\
& \left.\times\left[\left(1-x_{2}\right) \phi_{3}^{A}\left(x_{3}\right)+r_{3}\left(x_{3}-1\right)\left(\phi_{3}^{T}\left(x_{3}\right)+\phi_{3}^{P}\left(x_{3}\right)\right)\right] h_{n}\left(x_{1}, 1-x_{2}, 1-x_{3}, b_{1}, b_{2}\right)\right\} .
\end{aligned}
$$

$$
\begin{aligned}
F_{a n n}^{L L}\left(a_{i}\right)= & 8 \pi C_{F} m_{B}^{4} f_{B} \int_{0}^{1} d x_{2} d x_{3} \int_{0}^{\infty} b_{2} d b_{2} b_{3} d b_{3}\left\{a_{i}\left(t_{c}\right) E_{a}\left(t_{c}\right)\right. \\
& \times\left[-x_{3} \phi_{2}^{A}\left(x_{2}\right) \phi_{3}^{A}\left(x_{3}\right)-2 r_{2} r_{3}\left(1+x_{3}\right) \phi_{2}^{P}\left(x_{2}\right) \phi_{3}^{P}\left(x_{3}\right)\right. \\
& \left.+2 r_{2} r_{3}\left(1-x_{3}\right) \phi_{2}^{P}\left(x_{2}\right) \phi_{3}^{T}\left(x_{3}\right)\right] h_{a}\left(1-x_{2}, x_{3}, b_{2}, b_{3}\right) \\
& +\left[\left(1-x_{2}\right) \phi_{2}^{A}\left(x_{2}\right) \phi_{3}^{A}\left(x_{3}\right)+2 r_{2} r_{3}\left(2-x_{2}\right) \phi_{2}^{P}\left(x_{2}\right) \phi_{3}^{P}\left(x_{3}\right)+2 r_{2} r_{3} x_{2} \phi_{3}^{P}\left(x_{3}\right) \phi_{2}^{T}\left(x_{2}\right)\right] \\
& \left.\times a_{i}\left(t_{c}^{\prime}\right) E_{a}\left(t_{c}^{\prime}\right) h_{a}\left(x_{3}, 1-x_{2}, b_{3}, b_{2}\right)\right\}
\end{aligned}
$$

$$
F_{a n n}^{L R}\left(a_{i}\right)=F_{a n n}^{L L}\left(a_{i}\right),
$$

$$
\begin{aligned}
F_{a n n}^{S P}\left(a_{i}\right)= & 16 \pi C_{F} m_{B}^{4} f_{B} \int_{0}^{1} d x_{2} d x_{3} \int_{0}^{\infty} b_{2} d b_{2} b_{3} d b_{3}\left\{\left[2 r_{2} \phi_{2}^{P}\left(x_{2}\right) \phi_{3}^{A}\left(x_{3}\right)\right.\right. \\
& \left.+x_{3} r_{3} \phi_{2}^{A}\left(x_{2}\right)\left(\phi_{3}^{P}\left(x_{3}\right)-\phi_{3}^{T}\left(x_{3}\right)\right)\right] a_{i}\left(t_{c}\right) E_{a}\left(t_{c}\right) h_{a}\left(1-x_{2}, x_{3}, b_{2}, b_{3}\right) \\
& +\left[2 r_{3} \phi_{2}^{A}\left(x_{2}\right) \phi_{3}^{P}\left(x_{3}\right)+r_{2}\left(1-x_{2}\right)\left(\phi_{2}^{P}\left(x_{2}\right)+\phi_{2}^{T}\left(x_{2}\right)\right) \phi_{3}^{A}\left(x_{3}\right)\right] \\
& \left.\times a_{i}\left(t_{c}^{\prime}\right) E_{a}\left(t_{c}^{\prime}\right) h_{a}\left(x_{3}, 1-x_{2}, b_{3}, b_{2}\right)\right\} .
\end{aligned}
$$

$$
\begin{aligned}
M_{a n n}^{L L}\left(C_{i}\right)= & 32 \pi C_{F} m_{B}^{4} / \sqrt{6} \int_{0}^{1} d x_{1} d x_{2} d x_{3} \int_{0}^{\infty} b_{1} d b_{2} b_{2} d b_{2} \phi_{B}\left(x_{1}, b_{1}\right) \\
& \times\left\{h _ { n a } ( x _ { 1 } , 1 - x _ { 2 } , 1 - x _ { 3 } , b _ { 1 } , b _ { 2 } ) \left[\left(x_{2}-1\right) \phi_{2}^{A}\left(x_{2}\right) \phi_{3}^{A}\left(x_{3}\right)\right.\right. \\
& -r_{2} r_{3}\left(\left(x_{3}-1\right)\left(\phi_{2}^{P}\left(x_{2}\right)+\phi_{2}^{T}\left(x_{2}\right)\right)\left(\phi_{3}^{P}\left(x_{3}\right)-\phi_{3}^{T}\left(x_{3}\right)\right)+4 \phi_{2}^{P}\left(x_{2}\right) \phi_{3}^{P}\left(x_{3}\right)\right. \\
& \left.-x_{2}\left(\phi_{2}^{P}\left(x_{2}\right)-\phi_{2}^{T}\left(x_{2}\right)\right)\left(\phi_{3}^{P}\left(x_{3}\right)+\phi_{3}^{T}\left(x_{3}\right)\right)\right] C_{i}\left(t_{d}\right) E_{a}^{\prime}\left(t_{d}\right) \\
& +h_{n a}^{\prime}\left(x_{1}, 1-x_{2}, 1-x_{3}, b_{1}, b_{2}\right)\left[x_{3} \phi_{2}^{A}\left(x_{2}\right) \phi_{3}^{A}\left(x_{3}\right)\right. \\
& +r_{2} r_{3}\left(x_{3}\left(\phi_{2}^{P}\left(x_{2}\right)-\phi_{2}^{T}\left(x_{2}\right)\right)\left(\phi_{3}^{P}\left(x_{3}\right)+\phi_{3}^{T}\left(x_{3}\right)\right)\right. \\
& \left.\left.\left.+\left(1-x_{2}\right)\left(\phi_{2}^{P}\left(x_{2}\right)+\phi_{2}^{T}\left(x_{2}\right)\right)\left(\phi_{3}^{P}\left(x_{3}\right)-\phi_{3}^{T}\left(x_{3}\right)\right)\right)\right] C_{i}\left(t_{d}^{\prime}\right) E_{a}^{\prime}\left(t_{d}^{\prime}\right)\right\},
\end{aligned}
$$

$$
\begin{aligned}
M_{a n n}^{L R}\left(C_{i}\right)= & 32 \pi C_{F} m_{B}^{4} / \sqrt{6} \int_{0}^{1} d x_{1} d x_{2} d x_{3} \int^{\infty} b_{1} d b_{1} b_{2} d b_{2} \phi_{B}\left(x_{1}, b_{1}\right) \\
& \times\left\{h _ { n a } ( x _ { 1 } , 1 - x _ { 2 } , 1 - x _ { 3 } , b _ { 1 } , b _ { 2 } ) \left[r_{2}\left(1+x_{2}\right) \phi_{3}^{A}\left(x_{3}\right)\left(\phi_{2}^{P}\left(x_{2}\right)-\phi_{2}^{T}\left(x_{2}\right)\right)\right.\right. \\
& \left.+r_{3}\left(x_{3}-2\right) \phi_{2}^{A}\left(x_{2}\right)\left(\phi_{3}^{P}\left(x_{3}\right)+\phi_{3}^{T}\left(x_{3}\right)\right)\right] C_{i}\left(t_{d}\right) E_{a}^{\prime}\left(t_{d}\right) \\
& +h_{n a}^{\prime}\left(x_{1}, 1-x_{2}, 1-x_{3}, b_{1}, b_{2}\right)\left[r_{2}\left(1-x_{2}\right) \phi_{3}^{A}\left(x_{3}\right)\left(\phi_{2}^{P}\left(x_{2}\right)-\phi_{2}^{T}\left(x_{2}\right)\right)\right. \\
& \left.\left.-r_{3} x_{3} \phi_{2}^{A}\left(x_{2}\right)\left(\phi_{3}^{P}\left(x_{3}\right)+\phi_{3}^{T}\left(x_{3}\right)\right)\right] C_{i}\left(t_{d}^{\prime}\right) E_{a}^{\prime}\left(t_{d}^{\prime}\right)\right\}
\end{aligned}
$$




$$
\begin{aligned}
M_{a n n}^{S P}\left(C_{i}\right)= & 32 \pi C_{F} m_{B}^{4} / \sqrt{6} \int_{0}^{1} d x_{1} d x_{2} d x_{3} \int_{0}^{\infty} b_{1} d b_{1} b_{2} d b_{2} \phi_{B}\left(x_{1}, b_{1}\right) \\
& \times\left\{C _ { i } ( t _ { d } ) E _ { a } ^ { \prime } ( t _ { d } ) h _ { n a } ( x _ { 1 } , 1 - x _ { 2 } , 1 - x _ { 3 } , b _ { 1 } , b _ { 2 } ) \left[-x_{3} \phi_{2}^{A}\left(x_{2}\right) \phi_{3}^{A}\left(x_{3}\right)\right.\right. \\
& -4 r_{2} r_{3} \phi_{2}^{P}\left(x_{2}\right) \phi_{3}^{P}\left(x_{3}\right)+r_{2} r_{3}\left(1-x_{3}\right)\left(\phi_{2}^{P}\left(x_{2}\right)-\phi_{2}^{T}\left(x_{2}\right)\right)\left(\phi_{3}^{P}\left(x_{3}\right)+\phi_{3}^{T}\left(x_{3}\right)\right) \\
& \left.+r_{2} r_{3} x_{2}\left(\phi_{2}^{P}\left(x_{2}\right)+\phi_{2}^{T}\left(x_{2}\right)\right)\left(\phi_{3}^{P}\left(x_{3}\right)-\phi_{3}^{T}\left(x_{3}\right)\right)\right] \\
& +C_{i}\left(t_{d}^{\prime}\right) E_{a}^{\prime}\left(t_{d}^{\prime}\right) h_{n a}^{\prime}\left(x_{1}, 1-x_{2}, 1-x_{3}, b_{1}, b_{2}\right)\left[\left(1-x_{2}\right) \phi_{2}^{A}\left(x_{2}\right) \phi_{3}^{A}\left(x_{3}\right)\right. \\
& +r_{2} r_{3}\left(1-x_{2}\right)\left(\phi_{2}^{P}\left(x_{2}\right)-\phi_{2}^{T}\left(x_{2}\right)\right)\left(\phi_{3}^{P}\left(x_{3}\right)+\phi_{3}^{T}\left(x_{3}\right)\right) \\
& \left.\left.+r_{2} r_{3} x_{3}\left(\phi_{2}^{P}\left(x_{2}\right)+\phi_{2}^{T}\left(x_{2}\right)\right)\left(\phi_{3}^{P}\left(x_{3}\right)-\phi_{3}^{T}\left(x_{3}\right)\right)\right]\right\} .
\end{aligned}
$$

The hard scales involved in the above decay amplitudes are defined by

$$
\begin{aligned}
t_{a} & =\max \left\{\sqrt{1-x_{3}} m_{B}, 1 / b_{1}, 1 / b_{3}\right\}, \\
t_{a}^{\prime} & =\max \left\{\sqrt{x_{1}} m_{B}, 1 / b_{1}, 1 / b_{3}\right\}, \\
t_{b} & =\max \left\{\sqrt{x_{1}\left(1-x_{3}\right)} m_{B}, \sqrt{\left|x_{2}-x_{1}\right|\left(1-x_{3}\right)} m_{B}, 1 / b_{1}, 1 / b_{2}\right\}, \\
t_{b}^{\prime} & =\max \left\{\sqrt{x_{1}\left(1-x_{3}\right)} m_{B}, \sqrt{\left|1-x_{1}-x_{2}\right|\left(1-x_{3}\right)} m_{B}, 1 / b_{1}, 1 / b_{2}\right\}, \\
t_{c} & =\max \left\{\sqrt{x_{3}} m_{B}, 1 / b_{2}, 1 / b_{3}\right\}, \\
t_{c}^{\prime} & =\max \left\{\sqrt{1-x_{2}} m_{B}, 1 / b_{2}, 1 / b_{3}\right\}, \\
t_{d} & =\max \left\{\sqrt{\left(1-x_{2}\right) x_{3}} m_{B}, \sqrt{1-\left(x_{2}-x_{1}\right)\left(1-x_{3}\right)} m_{B}, 1 / b_{1}, 1 / b_{2}\right\}, \\
t_{d}^{\prime} & =\max \left\{\sqrt{x_{3}\left(1-x_{2}\right)} m_{B}, \sqrt{\left|x_{1}-\left(1-x_{2}\right)\right| x_{3}} m_{B}, 1 / b_{1}, 1 / b_{2}\right\} .
\end{aligned}
$$

The hard kernels $h$ in the decay amplitudes consist of two parts, the jet function $J_{t}\left(x_{i}\right)$ derived in the threshold resummation and the Fourier transformation of the virtual particle propagators:

$$
\begin{aligned}
h_{e}\left(x_{1}, x_{3}, b_{1}, b_{3}\right)= & {\left[\theta\left(b_{1}-b_{3}\right) I_{0}\left(\sqrt{x}_{3} m_{B} b_{3}\right) K_{0}\left(\sqrt{x_{3}} m_{B} b_{1}\right)\right.} \\
& \left.+\theta\left(b_{3}-b_{1}\right) I_{0}\left(\sqrt{x_{3}} m_{B} b_{1}\right) K_{0}\left(\sqrt{x_{3}} m_{B} b_{3}\right)\right] K_{0}\left(\sqrt{x_{1} x_{3}} m_{B} b_{1}\right) J_{t}\left(x_{3}\right),
\end{aligned}
$$

$$
\begin{aligned}
h_{n}\left(x_{1}, x_{2}, x_{3}, b_{1}, b_{2}\right)= & {\left[\theta\left(b_{2}-b_{1}\right) K_{0}\left(\sqrt{x_{1} x_{3}} m_{B} b_{2}\right) I_{0}\left(\sqrt{x_{1} x_{3}} m_{B} b_{1}\right)+\theta\left(b_{1}-b_{2}\right) K_{0}\left(\sqrt{x_{1} x_{3}} m_{B} b_{1}\right) I_{0}\left(\sqrt{x_{1} x_{3}} m_{B} b_{2}\right)\right] } \\
\times & \begin{cases}\frac{i \pi}{2} H_{0}^{(1)}\left(\sqrt{\left(x_{2}-x_{1}\right) x_{3}} m_{B} b_{2}\right), & x_{1}-x_{2}<0 \\
K_{0}\left(\sqrt{\left(x_{1}-x_{2}\right) x_{3}} m_{B} b_{2}\right), \quad x_{1}-x_{2}>0\end{cases} \\
h_{a}\left(x_{2}, x_{3}, b_{2}, b_{3}\right)= & \left(\frac{i \pi}{2}\right)^{2} J_{t}\left(x_{3}\right)\left[\theta\left(b_{2}-b_{3}\right) H_{0}^{(1)}\left(\sqrt{x_{3}} m_{B} b_{2}\right) J_{0}\left(\sqrt{x_{3}} m_{B} b_{3}\right)\right. \\
& \left.+\theta\left(b_{3}-b_{2}\right) H_{0}^{(1)}\left(\sqrt{x_{3}} m_{B} b_{3}\right) J_{0}\left(\sqrt{x_{3}} m_{B} b_{2}\right)\right] H_{0}^{(1)}\left(\sqrt{x_{2} x_{3}} m_{B} b_{2}\right) \\
& \\
h_{n a}\left(x_{1}, x_{2}, x_{3}, b_{1}, b_{2}\right)= & \frac{i \pi}{2}\left[\theta\left(b_{1}-b_{2}\right) H_{0}^{(1)}\left(\sqrt{x_{2}\left(1-x_{3}\right)} m_{B} b_{1}\right) J_{0}\left(\sqrt{x_{2}\left(1-x_{3}\right)} m_{B} b_{2}\right)\right. \\
& \left.+\theta\left(b_{2}-b_{1}\right) H_{0}^{(1)}\left(\sqrt{x_{2}\left(1-x_{3}\right)} m_{B} b_{2}\right) J_{0}\left(\sqrt{x_{2}\left(1-x_{3}\right)} m_{B} b_{1}\right)\right] \\
& \times K_{0}\left(\sqrt{1-\left(1-x_{1}-x_{2}\right) x_{3}} m_{B} b_{1}\right),
\end{aligned}
$$




$$
\begin{aligned}
h_{n a}^{\prime}\left(x_{1}, x_{2}, x_{3}, b_{1}, b_{2}\right)= & \frac{i \pi}{2}\left[\theta\left(b_{1}-b_{2}\right) H_{0}^{(1)}\left(\sqrt{x_{2}\left(1-x_{3}\right)} m_{B} b_{1}\right) J_{0}\left(\sqrt{x_{2}\left(1-x_{3}\right)} m_{B} b_{2}\right)\right. \\
& \left.+\theta\left(b_{2}-b_{1}\right) H_{0}^{(1)}\left(\sqrt{x_{2}\left(1-x_{3}\right)} m_{B} b_{2}\right) J_{0}\left(\sqrt{x_{2}\left(1-x_{3}\right)} m_{B} b_{1}\right)\right] \\
& \times\left\{\begin{array}{ll}
\frac{i \pi}{2} H_{0}^{(1)}\left(\sqrt{\left(x_{2}-x_{1}\right)\left(1-x_{3}\right)} m_{B} b_{1}\right), & x_{1}-x_{2}<0 \\
K_{0}\left(\sqrt{\left(x_{1}-x_{2}\right)\left(1-x_{3}\right)} m_{B} b_{1}\right), & x_{1}-x_{2}>0
\end{array},\right.
\end{aligned}
$$

with the Bessel function $H_{0}^{(1)}(z)=\mathrm{J}_{0}(z)+i \mathrm{Y}_{0}(z)$. The following approximate parametrization for the jet function has been proposed for convenience [38],

$$
J_{t}(x)=\frac{2^{1+2 c} \Gamma(3 / 2+c)}{\sqrt{\pi} \Gamma(1+c)}[x(1-x)]^{c},
$$

with the parameter $c \approx 0.3$. The prefactor in the above expression is chosen to obey the normalization $\int_{0}^{1} J_{t}(x) d x=1$. The jet function $J_{t}(x)$ gives a very small numerical effect to the nonfactorizable amplitude [42], so it is dropped from $h_{n}$ and $h_{n a}$.

The evolution factors $E_{e}^{(\prime)}$ and $E_{a}^{(\prime)}$ are written as

$$
\begin{array}{ll}
E_{e}(t)=\alpha_{s}(t) \exp \left[-S_{B}(t)-S_{3}(t)\right], & E_{e}^{\prime}(t)=\left.\alpha_{s}(t) \exp \left[-S_{B}(t)-S_{2}(t)-S_{3}(t)\right]\right|_{b_{1}=b_{3}}, \\
E_{a}(t)=\alpha_{s}(t) \exp \left[-S_{2}(t)-S_{3}(t)\right], & E_{a}^{\prime}(t)=\left.\alpha_{s}(t) \exp \left[-S_{B}(t)-S_{2}(t)-S_{3}(t)\right]\right|_{b_{2}=b_{3}},
\end{array}
$$

in which the Sudakov exponents are given by

$$
\begin{gathered}
S_{B}(t)=s\left(x_{1} \frac{m_{B}}{\sqrt{2}}, b_{1}\right)+\frac{5}{3} \int_{1 / b_{1}}^{t} \frac{d \bar{\mu}}{\bar{\mu}} \gamma_{q}\left(\alpha_{s}(\bar{\mu})\right), \\
S_{2}(t)=s\left(x_{2} \frac{m_{B}}{\sqrt{2}}, b_{2}\right)+s\left(\left(1-x_{2}\right) \frac{m_{B}}{\sqrt{2}}, b_{2}\right)+2 \int_{1 / b_{2}}^{t} \frac{d \bar{\mu}}{\bar{\mu}} \gamma_{q}\left(\alpha_{s}(\bar{\mu})\right),
\end{gathered}
$$

with the quark anomalous dimension $\gamma_{q}=-\alpha_{s} / \pi$. Replacing the kinematic variables of $M_{2}$ by those of $M_{3}$ in $S_{2}$, we get the expression for $S_{3}$. The function $s(Q, b)$ is expressed as

$$
\begin{aligned}
s(Q, b)= & \frac{A^{(1)}}{2 \beta_{1}} \hat{q} \ln \left(\frac{\hat{q}}{\hat{b}}\right)-\frac{A^{(1)}}{2 \beta_{1}}(\hat{q}-\hat{b})+\frac{A^{(2)}}{4 \beta_{1}^{2}}\left(\frac{\hat{q}}{\hat{b}}-1\right)-\left[\frac{A^{(2)}}{4 \beta_{1}^{2}}-\frac{A^{(1)}}{4 \beta_{1}} \ln \left(\frac{e^{2 \gamma_{E}-1}}{2}\right)\right] \ln \left(\frac{\hat{q}}{\hat{b}}\right) \\
& +\frac{A^{(1)} \beta_{2}}{4 \beta_{1}^{3}} \hat{q}\left[\frac{\ln (2 \hat{q})+1}{\hat{q}}-\frac{\ln (2 \hat{b})+1}{\hat{b}}\right]+\frac{A^{(1)} \beta_{2}}{8 \beta_{1}^{3}}\left[\ln ^{2}(2 \hat{q})-\ln ^{2}(2 \hat{b})\right], \\
& +\frac{A^{(1)} \beta_{2}}{8 \beta_{1}^{3}} \ln \left(\frac{e^{2 \gamma_{E}-1}}{2}\right)\left[\frac{\ln (2 \hat{q})+1}{\hat{q}}-\frac{\ln (2 \hat{b})+1}{\hat{b}}\right]-\frac{A^{(2)} \beta_{2}}{16 \beta_{1}^{4}}\left[\frac{2 \ln (2 \hat{q})+3}{\hat{q}}-\frac{2 \ln (2 \hat{b})+3}{\hat{b}}\right] \\
& -\frac{A^{(2)} \beta_{2}}{16 \beta_{1}^{4}} \frac{\hat{q}-\hat{b}}{\hat{b}^{2}}[2 \ln (2 \hat{b})+1]+\frac{A^{(2)} \beta_{2}^{2} \hat{q}-\hat{b}}{432 \beta_{1}^{6}} \frac{\hat{b}^{3}}{\hat{b}^{3}}\left[9 \ln ^{2}(2 \hat{b})+6 \ln (2 \hat{b})+2\right] \\
& +\frac{A^{(2)} \beta_{2}^{2}}{1728 \beta_{1}^{6}}\left[\frac{18 \ln ^{2}(2 \hat{q})+30 \ln (2 \hat{q})+19}{\hat{q}^{2}}-\frac{18 \ln ^{2}(2 \hat{b})+30 \ln (2 \hat{b})+19}{\hat{b}^{2}}\right],
\end{aligned}
$$

with the variables

$$
\hat{q} \equiv \ln \left[Q /\left(\sqrt{2} \Lambda_{\mathrm{QCD}}\right)\right], \quad \hat{b} \equiv \ln \left[1 /\left(b \Lambda_{\mathrm{QCD}}\right)\right],
$$

and the coefficients $A^{(i)}$ and $\beta_{i}$, 


$$
\begin{aligned}
\beta_{1} & =\frac{33-2 n_{f}}{12}, \quad \beta_{2}=\frac{153-19 n_{f}}{24}, \\
A^{(1)} & =\frac{4}{3}, \quad A^{(2)}=\frac{67}{9}-\frac{\pi^{2}}{3}-\frac{10}{27} n_{f}+\frac{8}{3} \beta_{1} \ln \left(\frac{1}{2} e^{\gamma_{E}}\right),
\end{aligned}
$$

where $n_{f}$ is the number of the quark flavors and $\gamma_{E}$ is the Euler constant. We adopt the one-loop running coupling constant, so only the first four terms of Eq. (A24) are picked up in the numerical analysis.
[1] S. Aoki et al. (Flavour Lattice Averaging Group), Eur. Phys. J. C 80, 113 (2020).

[2] R. Aaij et al. (LHCb Collaboration), Eur. Phys. J. C 73, 2373 (2013).

[3] E. Kou et al. (Belle-II Collaboration), Prog. Theor. Exp. Phys. (2019), 123C01; (2020), 029201(E).

[4] A. Cerri, V. V. Gligorov, S. Malvezzi, J. Martin Camalich, J. Zupan, S. Akar, J. Alimena, B. C. Allanach, W. Altmannshofer, L. Anderlini et al., CERN Yellow Rep. Monogr. 7, 867 (2019).

[5] P. Ball and R. Zwicky, Phys. Rev. D 71, 014015 (2005).

[6] P. Ball, V. M. Braun, and A. Lenz, J. High Energy Phys. 05 (2006) 004.

[7] P. Ball and G. W. Jones, J. High Energy Phys. 03 (2007) 069.

[8] G. S. Bali et al. (RQCD Collaboration), Phys. Lett. B 774, 91 (2017).

[9] G. S. Bali et al. (QCD Collaboration), J. High Energy Phys. 08 (2019) 065; 11 (2020) A037.

[10] J. Hua, M. H. Chu, P. Sun, W. Wang, J. Xu, Y. B. Yang, J. H. Zhang, and Q. A. Zhang, arXiv:2011.09788.

[11] V. L. Chernyak and I. R. Zhitnitsky, Nucl. Phys. B345, 137 (1990).

[12] A. Ali, V. M. Braun, and H. Simma, Z. Phys. C 63, 437 (1994).

[13] A. Khodjamirian, Nucl. Phys. B605, 558 (2001).

[14] M. Beneke, G. Buchalla, M. Neubert, and C. T. Sachrajda, Phys. Rev. Lett. 83, 1914 (1999).

[15] H. n. Li and H. L. Yu, Phys. Rev. Lett. 74, 4388 (1995).

[16] H. n. Li and H. L. Yu, Phys. Lett. B 353, 301 (1995).

[17] H. n. Li and H. L. Yu, Phys. Rev. D 53, 2480 (1996).

[18] Y. Y. Keum and H. n. Li, Phys. Rev. D 63, 074006 (2001).

[19] Y. Y. Keum, H. n. Li, and A. I. Sanda, Phys. Rev. D 63, 054008 (2001).

[20] C. D. Lu, K. Ukai, and M. Z. Yang, Phys. Rev. D 63, 074009 (2001).

[21] C. W. Bauer, S. Fleming, and M. E. Luke, Phys. Rev. D 63, 014006 (2000).

[22] C. W. Bauer, S. Fleming, D. Pirjol, and I. W. Stewart, Phys. Rev. D 63, 114020 (2001).

[23] H. D. Li, C. D. Lü, C. Wang, Y. M. Wang, and Y. B. Wei, J. High Energy Phys. 04 (2020) 023.

[24] T. Huber, S. Kränkl, and X. Q. Li, J. High Energy Phys. 09 (2016) 112.

[25] G. Bell, M. Beneke, T. Huber, and X. Q. Li, J. High Energy Phys. 04 (2020) 055.

[26] P. A. Zyla et al. (Particle Data Group), Prog. Theor. Exp. Phys. (2020), 083C01.
[27] M. K. Jones et al. (Jefferson Lab Hall A Collaboration), Phys. Rev. Lett. 84, 1398 (2000).

[28] O. Gayou et al. (Jefferson Lab Hall A Collaboration), Phys. Rev. Lett. 88, 092301 (2002).

[29] J. P. Ralston and P. Jain, Phys. Rev. D 69, 053008 (2004).

[30] S. Nandi and H. n. Li, Phys. Rev. D 76, 034008 (2007).

[31] J. Botts and G. F. Sterman, Nucl. Phys. B325, 62 (1989).

[32] H. n. Li and G. F. Sterman, Nucl. Phys. B381, 129 (1992).

[33] H. n. Li, Nucl. Phys. A684, 304 (2001).

[34] A. Szczepaniak, E. M. Henley, and S. J. Brodsky, Phys. Lett. B 243, 287 (1990).

[35] G. Burdman and J. F. Donoghue, Phys. Lett. B 270, 55 (1991).

[36] M. Beneke and T. Feldmann, Nucl. Phys. B592, 3 (2001).

[37] J. C. Collins and D. E. Soper, Nucl. Phys. B193, 381 (1981); B213, 545(E) (1983).

[38] T. Kurimoto, H. n. Li, and A. I. Sanda, Phys. Rev. D 65, 014007 (2001).

[39] Z. T. Wei and M. Z. Yang, Nucl. Phys. B642, 263 (2002).

[40] R. Akhoury, G. F. Sterman, and Y. P. Yao, Phys. Rev. D 50, 358 (1994).

[41] G. P. Korchemsky, D. Pirjol, and T. M. Yan, Phys. Rev. D 61, 114510 (2000).

[42] H. n. Li, Phys. Rev. D 66, 094010 (2002).

[43] Z. Q. Zhang and H. n. Li, Eur. Phys. J. C 81, 595 (2021).

[44] H. n. Li, Y. L. Shen, and Y. M. Wang, J. High Energy Phys. 01 (2014) 004.

[45] G. Buchalla, A. J. Buras, and M. E. Lautenbacher, Rev. Mod. Phys. 68, 1125 (1996).

[46] M. Beneke, G. Buchalla, M. Neubert, and C. T. Sachrajda, Nucl. Phys. B591, 313 (2000).

[47] M. Beneke, G. Buchalla, M. Neubert, and C. T. Sachrajda, Nucl. Phys. B606, 245 (2001).

[48] C. H. V. Chang and H. n. Li, Phys. Rev. D 55, 5577 (1997).

[49] T. W. Yeh and H. n. Li, Phys. Rev. D 56, 1615 (1997).

[50] H. Y. Cheng, H. n. Li, and K. C. Yang, Phys. Rev. D 60, 094005 (1999).

[51] H. n. Li, arXiv:hep-ph/0110365.

[52] A. Ali, G. Kramer, Y. Li, C. D. Lu, Y. L. Shen, W. Wang, and Y. M. Wang, Phys. Rev. D 76, 074018 (2007).

[53] P. Lepage and C. Gohlke, gplepage/lsqfit: lsqfit version 11.7, Zenodo https://doi.org/10.5281/zenodo.4037174.

[54] G. S. Bali et al. (RQCD Collaboration), J. High Energy Phys. 08 (2019) 065.

[55] V. M. Braun, P. C. Bruns, S. Collins, J. A. Gracey, M. Gruber, M. Göckeler, F. Hutzler, P. Pérez-Rubio, A. Schäfer, W. Söldner et al., J. High Energy Phys. 04 (2017) 082.

[56] H. M. Choi and C. R. Ji, Phys. Rev. D 75, 034019 (2007). 
[57] R. Aaij et al. (LHCb Collaboration), Phys. Rev. Lett. 126, 091802 (2021).

[58] H. n. Li and S. Mishima, Phys. Rev. D 74, 094020 (2006).

[59] Z. Rui, X. Gao, and C. D. Lu, Eur. Phys. J. C 72, 1923 (2012).

[60] H. n. Li, Y. L. Shen, and Y. M. Wang, Phys. Rev. D 85, 074004 (2012).

[61] W. F. Wang and Z. J. Xiao, Phys. Rev. D 86, 114025 (2012).

[62] Y. Yang, L. Lang, X. Zhao, J. Huang, and J. Sun, Phys. Rev. D 103, 056006 (2021).
[63] S. H. Zhou and C. D. Lü, Chin. Phys. C 44, 063101 (2020).

[64] H. Y. Cheng, arXiv:2005.06080.

[65] Y. Li, A. J. Ma, W. F. Wang, and Z. J. Xiao, Phys. Rev. D 95, 056008 (2017).

[66] C. D. Lu and M. Z. Yang, Eur. Phys. J. C 23, 275 (2002).

[67] D. C. Yan, P. Yang, X. Liu, and Z. J. Xiao, Nucl. Phys. B931, 79 (2018).

[68] Y. M. Wang and Y. L. Shen, J. High Energy Phys. 12 (2017) 037.

[69] Y. M. Wang and Y. L. Shen, J. High Energy Phys. 05 (2018) 184. 